\title{
Amygdala subnuclei response and connectivity during emotional processing
}

Stanislau Hrybouski ${ }^{1}$, Arash Aghamohammadi-Sereshki*1, Christopher R. Madan*2,5, Andrea T. Shafer* ${ }^{1,6}$, Corey A. Baron ${ }^{3}$, Peter Seres ${ }^{3}$, Christian Beaulieu ${ }^{3}$, Fraser Olsen ${ }^{1,3}$, Nikolai V. Malykhin ${ }^{1,3,4}$

*Authors with equal contributions.

${ }^{1}$ Neuroscience and Mental Health Institute, University of Alberta, Edmonton, AB, Canada, T6G 2E1

${ }^{2}$ Department of Psychology, University of Alberta, Edmonton, AB, Canada, T6G 2E9

${ }^{3}$ Department of Biomedical Engineering, University of Alberta, Edmonton, AB, Canada, T6G 2V2

${ }^{4}$ Department of Psychiatry, University of Alberta, Edmonton, AB, Canada, T6G 2B7

${ }^{5}$ Department of Psychology, Boston College, Chestnut Hill, MA, USA, 02467

${ }^{6}$ Institute of Gerontology, Wayne State University, Detroit, MI, USA, 48202

\section{Corresponding author:}

Nikolai V. Malykhin, M.D., Ph.D.

University of Alberta

Dept. of Biomedical Engineering

\#1069, Research Transition Facility

University of Alberta, AB, Canada

T6G 2V2

Phone: (780) 248-1120

Email: nikolai@ualberta.ca 


\begin{abstract}
The involvement of the human amygdala in emotion-related processing has been studied using functional magnetic resonance imaging (fMRI) for many years. However, despite the amygdala being comprised of several subnuclei, most studies investigated the role of the entire amygdala in processing of emotions. Here we combined a novel anatomical tracing protocol with eventrelated high-resolution fMRI acquisition to study the responsiveness of the amygdala subnuclei to negative emotional stimuli and to examine intra-amygdala functional connectivity. The greatest sensitivity to negative emotional stimuli was observed in the centromedial amygdala, where the hemodynamic response amplitude elicited by negative emotional stimuli was greater and peaked later than for neutral stimuli. Connectivity patterns converge with extant findings in animals, such that the strongest connectivity was between the lateral nucleus and the nuclei of the basal amygdala. Current findings provide evidence of functional specialization within the human amygdala.
\end{abstract}

Keywords: high-resolution fMRI, amygdala subnuclei, amygdala connectivity, emotions

\title{
Introduction
}

The amygdala $(\mathrm{AG})$ is a fundamental structure involved in the neural circuits of fear and reward learning, as well as aggressive, sexual, maternal, and feeding behaviours (Janak and Tye, 2015; LeDoux, 2012; LeDoux and Schiller, 2009). Through extensive interactions with cortical and other subcortical areas, the AG also modulates attention and perception (Adolphs et al., 2005; Pitkänen et al., 2000; Sah et al., 2003). Of particular interest is the AG's involvement in processing of emotionally relevant stimuli and encoding of emotionally salient memories 
(Dolcos et al., 2004; Kensinger, 2009; LeDoux and Schiller, 2009; Murty et al., 2010; Sergerie et al., 2008; Shafer et al., 2012; Shafer and Dolcos, 2012).

To gain a better understanding of the functional role of the AG, it is important to acknowledge that the $\mathrm{AG}$ is not a homogenous structure and thus it is crucial to differentiate response properties of the AG subnuclei (LeDoux, 2000, 2012; Sah et al., 2003). Human and animal studies have demonstrated that the AG is a gray matter complex, composed of at least thirteen distinct nuclei (Brabec et al., 2010; Janak and Tye, 2015; LeDoux, 2012; Sah et al., 2003; Whalen et al., 2009). These are generally grouped into two major subdivisions: basolateral and centrocorticomedial (Johnston, 1923; LeDoux and Schiller, 2009). Lateral (LA), basal (B), and accessory basal (AB) nuclei constitute the basolateral (BLA) complex, while cortical (Co), medial (M), and central (Ce) nuclei belong to the centrocorticomedial group.

For over fifty years, the AG has been thought to be fundamental to processing of emotions (Weiskrantz, 1956), with much of the non-human research utilizing Pavlovian conditioning procedures, a form of emotional learning in which a biologically irrelevant stimulus starts to elicit defensive behaviors, and physiological responses when associated with an aversive or threatening event (Davis and Whalen, 2001; LeDoux, 2000, 2012; Phelps and LeDoux, 2005). Animal fear conditioning experiments demonstrated that the nuclei of the AG play unique roles in this form of learning (LeDoux 2012; Macedo et al., 2007; Phelps and LeDoux, 2005).

Human fMRI and patient studies proved that the human AG is also involved in fear conditioning (Bach et al., 2011; Büchel et al., 1998; Klumpers et al., 2014; LaBar et al., 1998; Phelps et al., 2004). Furthermore, recent studies of patients with Urbach-Wiethe disease (UWD) support the notion of functional specialization of the AG subnuclei. For instance, focal lesions to the BLA 
AG result in reduced fear conditioning (Klumpers et al., 2014), unregulated fear hypervigilance (Terburg et al., 2012), and extreme generosity (van Honk et al., 2013). This is in contrast to studies of a UWD patient with bilateral loss of the entire AG, who is incapable of recognizing fear in facial expressions (Adolphs et al., 1994, 1995). Despite these advances in our understanding of the human AG, it is still poorly understood how various AG subnuclei contribute to processing of emotional information in a broader context.

To understand functional significance of the AG subnuclei, it is crucial to understand how neurons within the AG subnuclei interact with each other. In rodent literature, there is substantial debate between two potential mechanisms of intra-AG communication: validity of the classical serial model is questioned by the proponents of the parallel processing model. The serial model proposes that intra-AG information-processing stream occurs predominantly from the LA nucleus to the $\mathrm{BA} / \mathrm{AB}$ nuclei, and from the $\mathrm{BA} / \mathrm{AB}$ nuclei to the Ce nucleus (Duvarci and Paré, 2014; Freese and Amaral, 2009; Pitkänen et al. 1997). However, numerous animal reward learning studies revealed competing functions of the CeM and the BLA AG (for review see Balleine and Killcross, 2006). Although some attempts have been made to investigate the AG connectivity in humans using fMRI (Grant, et al., 2015; Roy, et al., 2009), these studies were limited by low spatial resolution in echo planar imaging. Consequently, little is known about how the human AG subnuclei interact with each other.

Although most fMRI acquisition sequences do not provide sufficient spatial resolution to identify individual subnuclei of the AG, several authors have coarsely segregated AG activations into dorsal vs. ventral (Kim et al., 2004; Morris et al., 2001; Whalen et al., 1998; Whalen et al., 2001), medial vs. lateral (Kim et al., 2003; Zald and Pardo, 2002), and anterior vs. posterior (Gottfried et al., 2002; Morris et al., 2002; Wang et al., 2008) subdivisions. More recent human 
fMRI studies, with coronal plane resolution of 4- $\mathrm{mm}^{2}$ or less, and total voxel volume of $8-\mathrm{mm}^{3}$ or less, attempted to localize AG activations more precisely (see Table 1). Most of these studies employed image-processing pipelines, which relied on normalizing participants' MR images to a common template space (i.e., MNI or Talairach). Unfortunately, recent evidence indicates that such procedures detect not only hemodynamic changes within the AG, but are also substantially influenced by activity in more distal brain regions (see Boubela et al., 2015). Furthermore, there are substantial variations in methodology used to localize intra-AG activations. Generally, two main references have been used: Mai (2008) anatomical atlas (Bach et al., 2011; Boll et al., 2011, 2013; Gamer et al., 2010; Prévost et al., 2011, 2012, 2013) or Amunts et al. (2005) probabilistic atlas of the human AG subnuclei (Ball et al. 2007, 2009; Frühholz and Grandjean, 2013; Grant et al, 2015; Roy et al., 2009; Styalidis et al., 2014).

Because of substantial methodological differences between human and animal studies of the AG there is a notable discrepancy between our knowledge of the AG subnuclei function in humans and animals. To bridge this gap, we defined human emotion as a psychological response driven by neurophysiological survival and reinforcement circuits (see LeDoux, 2012, 2014, for comprehensive reviews), and relied on the framework established by the core-affect model (Russell, 1980, 2003) to elicit AG responses. The core affect theory proposes that, at a fundamental level, emotional information is interpreted as a blend of two independent dimensions - pleasantness and intensity (Bradley \& Lang, 1994; Russell, 1980, 2003). These dimensions are consistent across cultures, and can be used to describe an individual's affective response to verbal and non-verbal stimuli, facial expressions, sounds, body postures, and odors (e.g., Bradley \& Lang 1994; Lang et al., 1998; Russell 1980, 2003). In combination with other 
factors, such as context, cultural experience, and genetic makeup, arousal and valence dimensions contribute to the human experience of feelings (Barret, 2006; Russell, 2003).

The primary goal of this study was to investigate sensitivity of the human AG subnuclei to high arousing negative emotion. Our secondary goal was to understand how the human AG subnuclei might function at the network level. To answer these questions we acquired high-resolution structural and functional MRI data at high field, and manually subdivided each participant's AG into 3 subnuclei groups. To improve accuracy and validity of our BOLD response measurements, we used a two-parameter hemodynamic response function in the fMRI analysis procedure, which allowed not only response amplitude, but also response timing (delay to peak) to explain the effects of emotional processing on the AG subnuclei groups. To answer our secondary research question, we examined intra-AG functional connectivity by assessing relationships between the subnuclei group time courses.

\section{Material and Methods}

\section{Participants}

A total of 28 right-handed healthy volunteers were recruited through online, newspaper, and poster advertisements. Healthy volunteers had no lifetime psychiatric disorders and no reported psychosis or mood disorders in first-degree relatives, as assessed by the Anxiety Disorders Interview Schedule-IV (Brown et al., 2004; Di Nardo et al., 1994), which provides detailed assessment of several anxiety disorders, affective disorders, and substance use disorders. The project's participants had no history of medical, neurological disorders, and had no prior exposure to the stimuli used in the fMRI paradigm. Medical exclusion criteria were defined as those active and inactive medical conditions that may interfere with normal cognitive function: 
cerebrovascular pathology, all tumors or congenital malformations of the nervous system, diabetes, multiple sclerosis, Parkinson's disease, epilepsy, dementia, organic psychosis (other than dementia), schizophrenia, and stroke. Medications that directly affect cognition, including alcohol, anti-cholinergic medications, benzodiazepines, antipsychotics, and antidepressants were also exclusionary. Written, informed consent was obtained from each participant. Imaging data from three participants was excluded from analyses due to excessive head motion, reducing the sample size to 25 individuals ( 12 females) with mean age of 27.6 years $(19-46, \mathrm{SD}=6.2)$. This study was approved by the University of Alberta Health Research Ethics Board.

\section{Stimuli}

Pictures were selected from the International Affective Picture System (IAPS; Lang et al., 2008) database based on normative ratings for valence and arousal (see Supplementary Materials for IAPS codes) and were supplemented with in-house pictures used in prior studies (Singhal et al., 2012; Wang et al., 2005, 2008). All pictures contained a central biological presence (predominantly a head/face). The chosen pictures were grouped into four categories (15 pictures/category) based on normative scores and ratings scores from a separate pilot dataset $(\mathrm{N}=8)$. Mean valence/arousal scores (9-point scale, as described below) were as follows: Neutral (Neu: 5.8/1.6), Low Emotional (Low Emo: 3.6/3.3), Medium Emotional (Med Emo: 2.3/5.8), and High Emotional (High Emo: 2.3/6.1). A repeated-measure ANOVA showed that valence ratings for each category were significantly different from each adjacent category except for Med and High Emo (i.e., Neu $>$ Low Emo $>$ Med Emo $=$ High Emo, $[F(3,72)=132.97, p<.001$, $\left.\left.\eta^{2}=.85\right]\right)$. A repeated-measure ANOVA of arousal ratings showed that each category was significantly different from each adjacent category such that, Neu $<$ Low Emo $<$ Medium Emo $<$ 
High Emo $\left[F(3,72)=150.59, p<.001, \eta^{2}=.86\right]$. Pair-wise comparisons were HolmBonferroni-corrected.

\section{Experimental Design}

Pictures were partitioned across five experimental runs such that three pictures per category were presented in each run. To prevent fatigue, participants received a break between experimental runs. Each picture was presented only once for $2 \mathrm{~s}$ and was followed by a response screen for $2 \mathrm{~s}$. The inter-trial interval was randomized on a negative exponential distribution with a median of 8 $\mathrm{s}$, ranging from 6 to $14 \mathrm{~s}$. To attenuate habituation, pictures were presented to participants in a semi-random sequence, such that no more than two pictures of the same category were shown sequentially. Participants were instructed to categorize their emotional reaction to each picture on a 4-point scale by pressing one of $4 \mathrm{MR}$-compatible buttons ( $1=$ 'non-emotional', 4 = 'very emotional'). After the scanning session, participants rated the pictures viewed in the scanner on valence and arousal using the 9-point Self-Assessment Manikin scale (Bradley and Lang, 1994).

\section{Data acquisition}

All images were acquired on a 4.7T Varian Inova MRI scanner at the Peter Allen MR Research Centre (University of Alberta, Edmonton, AB). 370 functional volumes were collected axially (in parallel to the AC-PC line) over 5 runs using a custom-written T2*-sensitive Gradient Echo Planar Imaging (EPI) pulse sequence [repetition time (TR): $2000 \mathrm{~ms}$; echo time (TE): $19 \mathrm{~ms}$; flip angle: $90^{\circ}$; field of view (FOV): $168 \times 210 \mathrm{~mm}^{2}$; voxel size: $1.5 \times 1.5 \times 1.4 \mathrm{~mm}^{3}$; interslice gap: 0.1 mm; 35 slices; GRAPPA parallel imaging with acceleration factor 2 (Griswold et al., 2002)]. For the AG segmentation and subdivision, high-resolution coronal structural images were acquired perpendicular to the AC-PC orientation using a custom-written T2-weighted 2D Fast Spin Echo (FSE) sequence [TR: $1100 \mathrm{~ms}$; TE: $39 \mathrm{~ms}$; FOV: $200 \times 200 \mathrm{~mm}^{2}$; voxel size: $0.52 \times 0.68 \times 1.0 \mathrm{~mm}^{3}$; 
90 slices]. To improve image registration accuracy between the anatomical scans and the functional data, axial high-resolution 2D FSE images were also acquired with coverage closely matching the functional data [TR: $7000 \mathrm{~ms}$; TE: $38 \mathrm{~ms}$; FOV: $210 \times 200 \mathrm{~mm}^{2}$; voxel size: $0.52 \times 0.68 \times 1.0 \mathrm{~mm}^{3} ; 45$ slices]. A whole brain T1-weighted 3D Magnetization Prepared Rapid Gradient-Echo (MPRAGE) sequence [TR: $8.5 \mathrm{~ms}$; TE: $4.5 \mathrm{~ms}$; inversion time: $300 \mathrm{~ms}$; flip angle: $10^{\circ}$; FOV: $256 \times 200 \times 180 \mathrm{~mm}^{3}$; voxel size: $1 \times 1 \times 1 \mathrm{~mm}^{3}$ ] was used to acquire anatomical images for automatic tissue segmentation. Fig. 1 demonstrates orientation and brain tissue coverage for structural and functional data.

\section{Amygdala Segmentation and Subdivision}

In agreement with some previous studies (Entis et al., 2012; Prévost et al., 2011), we found that the Amunts et al. (2005) AG map extends beyond the limits of the AG into the MTL white matter suggesting a mismatch between the anatomical location of the AG in our sample, and its location according to the Amunts et al. (2005) atlas. Because of this limitation, and because source localization using probabilistic maps necessitates manipulation of MRI data into standard space, a process that can produce inaccuracies due to the deformations required (Yassa and Stark, 2009), we used Mai (2008) atlas to manually segment the AG and its subnuclei groups in native space.

All AG ROIs were traced with a mouse-driven cursor using the interactive freely available software program Freeview v. 4 (MGH, Boston, MA). The AG was traced on each participants' high-resolution structural scan by the developer of the protocol (NM), who has extensive experience with the method for which a comprehensive description has been previously published (Malykhin et al., 2007). Next, a single rater (AAS) subdivided each AG into three subnuclei groups: (1) the centromedial (CeM) group, consisting of the Ce and the M nuclei; (2) 
the basal (BA) group, consisting of the $\mathrm{B}$, the AB, and the Co nuclei; and (3) the LA nucleus (Figs. 2 a-h). Though our intention was to measure BOLD signal from all of the principle AG subnuclei, due to technical limitations of the fMRI acquisition we were able to delineate and measure BOLD signal only from three major subnuclei groups. Many previous studies combined the $\mathrm{B}$, the $\mathrm{AB}$, and the LA nuclei into a single functional unit, the BLA complex; however, Boll et al. (2011) provided evidence that in humans these nuclei might respond differently in certain tasks. Furthermore, animal experiments demonstrated that the LA nucleus and the B/AB nuclei display distinct neurophysiological and connectivity properties (Duvarci and Paré, 2014; Janak et al., 2015). For these reasons, we studied the nuclei of the BA group separately from the LA nucleus.

Since macroscopic delineation of the AG subnuclei requires histological staining, we divided the human AG into subregions corresponding to approximations of the CeM, BA, and LA subnuclei groups. First we outlined global AG boundaries on sagittal, axial, and coronal planes as was previously described by Malykhin et al. (2007). Our measurements started from the most posterior slice of the AG (Fig. 2b), continued through slices where both the AG and the hippocampus were present, and ended at the level of the lateral sulcus closure. Once the total AG boundaries were outlined, only the coronal plane was used to subdivide the AG into subnuclei groups using a single internal landmark line, defined on each coronal slice where the AG is present. This landmark line allowed us to separate the AG into subnuclei groups, approximately matching the intra-AG anatomy described in the Mai (2008) atlas, and a postmortem histological study by Brabec et al. (2010).

First, we delineated the CeM group. Next, the LA nucleus was defined. Subsequently, the remaining AG tissue was assigned to the BA group. Our approach relied on one principle 
landmark line. Initially, this line is horizontal, and was drawn by connecting the most medial border of the AG with the most lateral border of the AG (Fig. 2c,d). As soon as the AG expanded in the inferior-lateral direction, the landmark line was drawn by connecting the most inferior medial border of the AG with the most medial inferior border of the AG (Fig. 2e-g). On each slice of the AG, we placed point A exactly in the middle of the landmark line (see Fig. 2cg). On slices, where the hippocampus is present the landmark line was also split into the lateral $1 / 3$ and the medial $2 / 3$ by point B, (see Fig. 2d,e). These two points were then used to define two secondary lines. The first line, subsequently called 'line A,' began on the AG border, directly above point $\mathrm{A}$, and was drawn at a $45^{\circ}$ angle (from the horizontal plane) towards the lateral border of the AG (Fig. 2c-g). The second line, subsequently called 'line B,' began on the AG border, directly above point $\mathrm{B}$, and was also drawn at a $45^{\circ}$ angle (from the horizontal plane) towards the lateral border of the AG (Fig. 2d,e).

Measurements of the CeM group started at the first coronal slice, when the AG appears as a small grey matter structure superior to the uncal recess (Malykhin et al., 2007; Fig. 2b). The CeM group occupies the entirety of the AG tissue until the AG extends (completely or partially) towards the ambient gyrus (Fig. 2b). In subsequent slices, the CeM group occupies only the superior portion of the AG, separated from the BA group by a horizontal line, drawn from the intersection of line A with the lateral border of the AG towards the medial border of the AG (Fig. 2c-f). This rule was used to define the inferior border of the CeM group on all slices anterior to the AG extension towards the ambient gyrus. In general, the last slice of the $\mathrm{CeM}$ group was $3 \mathrm{~mm}$ ( 3 slices) anterior to the most anterior slice of the hippocampus (Mai et al., 2008; Fig. 2a,g). 
The LA nucleus usually starts $2 \mathrm{~mm}$ (2 slices) anterior to the AG extension towards the ambient gyrus (Mai, 2008; Fig. 2d). Since the LA nucleus occupies the inferior-lateral portion of the AG (Fig. 2d-g), our goal was to establish its superior-medial border, which separates the LA nucleus from the BA group. Prior to the disappearance of the hippocampus, this was accomplished using line B (Fig. 2d,e). When the hippocampus is no longer present, the LA nucleus extends medially and line A was used to define the superior-medial boundary of the LA nucleus.

Once the CeM group and the LA nucleus were demarcated, the remaining AG tissue was assigned to the BA group (Fig. 2f). In the most anterior slices, where the CeM group is not present, the AG consists of the BA group and the LA nucleus only (Mai et al., 2008; Fig. 2a,g). In the last (i.e. most anterior) slice, all of the AG tissue was assigned to the LA nucleus (Fig. 2h). ITK-SNAP (v. 3.2.0; Yushkevich et al., 2006) was used to construct 3D models of these subnuclei groups (Fig. 3).

The AG subnuclei group ROIs were resampled to fMRI resolution and manually inspected for overlap with major blood vessels and susceptibility artifacts. ROI voxels corresponding to blood vessels and signal drop-outs were excluded from statistical analyses.

Reliability for the total AG and its subnuclei measurements was assessed by retracing the AG from 5 participants (10 AG total) after a one-week delay. Inter/intra-rater reliability intra-class correlations coefficients (ICCs) for the AG subnuclei were 0.90/0.85 for the CeM group, 0.90/0.94 for the LA nucleus, 0.98/0.96 for the BA group, and 0.97/0.95 for the total AG.

\section{Image Preprocessing}

Most of the image processing was performed in SPM8 (Wellcome Trust Centre for Neuroimaging, UCL, UK). Prior to registration, MPRAGE images underwent correction for 
intensity non-uniformity using N3 (McGill University, Montreal, QC). Due to differences in coverage between three anatomical scans, anatomical images were cropped using ImageJ $(\mathrm{NIH}$, Bethesda, MD) to isolate areas of overlapping coverage. Subsequently, overlapping portions of anatomical images were registered to each other using automated rigid-body transformations.

Next, the first functional volume was registered to the axial FSE image using a combination of manual and automatic registration tools. Functional data was then realigned to the first volume and corrected for slice acquisition delay. Artifact Detection Tool (ART; http://www.nitrc.org/projects/artifact_detect/) was used to identify signal spikes and to account for spin-history-related head-movement artifacts in the fMRI time series. The head-movement threshold was set at $0.5 \mathrm{~mm} / \mathrm{TR}$, and signal intensity threshold was set at 3 SDs from the global signal mean. Volumes that exceeded these thresholds were excluded (5 volumes/run, on average). No spatial smoothing was applied to the functional data.

White matter (WM) and cerebrospinal fluid (CSF) masks were thresholded at 0.90 tissue probability and resampled to functional volume coverage and resolution. Voxels classified as WM and CSF were used as signal sources of no-interest. As our goal was to account for as much physiological noise as possible without substantially sacrificing statistical power, we extracted the first five principal eigenvariates from the raw WM signal and the first five principle eigenvariates from the raw CSF signal using REX toolbox (http://www.nitrc.org/projects/rex/). Effects of motion were co-varied out of WM and CSF time courses, and the filtered WM and CSF signal eigenvariates were used as regressors of no-interest together with 6 head motion parameters in the General Linear Model (GLM) analysis. In all GLM procedures low frequency signal drifts were removed with a high-pass filter (128 s), and AR1 correction for serial autocorrelation was applied. 
To ensure that BOLD sensitivity was consistent across all AG ROIs, we computed temporal signal-to-noise ratio (tSNR) for each AG subnuclei group, averaged across all voxels in each ROI. Left/right tSNR for the CeM group was 14.9/16.4 $(\mathrm{SD}=2.1 / 2.4), 13.7 / 15.2(\mathrm{SD}=2.0 / 2.1)$ for the BA group, and 13.6/15.2 $(\mathrm{SD}=1.8 / 1.7)$ for the LA nucleus. This demonstrated that signal profiles were similar for all the AG subnuclei groups.

\section{General Linear Model and Estimation of Response Amplitude and Delay-to-Peak}

The profile of the hemodynamic response function in the AG may not be identical to a standard double-gamma function often used to model cortical responses (Devonshire et al., 2012; Handwerker et al., 2004; Pernet, 2014). Extracting the raw percentage of signal change over points in time after stimulus onset without reference to a standard hemodynamic template often leads to more accurate BOLD signal measurements. Unfortunately, this approach also weakens statistical power and can lead to overfitting. Here, we introduce an approach that minimizes the hemodynamic response function (HRf) bias, without substantial loss of statistical power.

The HRf was deconvolved using a Finite Impulse Response (FIR) model as implemented in MarsBar (v. 0.43; http://marsbar.sourceforge.net) toolbox. Events for all picture categories were pooled together and the mean HRf was estimated for each hemisphere's AG, separately for each participant. The fitted HRf timecourses were then averaged across all participants and hemispheres.

Next, we fit a double-gamma function (as implemented in the spm_hrf function within SPM) to the mean fitted time course using the SIMPLEX algorithm (Nelder and Mead, 1965). Five parameters (delay to response, delay of undershoot, dispersion of response, dispersion of undershoot, and ratio of response to undershoot) were optimized over 15,000 iterations to 
minimize the root-mean-squared-deviation (RMSD) between the double-gamma function and the FIR-fitted HRf time course (Fig. 4). The optimized double-gamma model, along with its firstorder derivative, was used to estimate the BOLD response for each stimulus category. MarsBar was used to extract HRf and time derivative betas for each event type from each ROI. In total, each picture category was represented by 15 trials/HRfs.

The BOLD response amplitude at peak was estimated using a protocol proposed by Calhoun et al. (2004). To resolve the ambiguous-sign cases of response amplitude (i.e., activation or deactivation) we developed a method that utilizes group-level parameter estimates to resolve the ambiguous cases of single-subject parameter estimates (see Supplementary Materials). Once the sign of each ambiguous subject-level parameter was resolved, we were able to compute subjectlevel delay-to-peak response values by finding the time-point of either maximum activation or maximum deactivation. For simplicity of interpretation, final response amplitude estimates were rescaled to percent signal change units using MarsBar toolbox.

To ensure that neither the BOLD response amplitude, nor the delay-to-peak information used in the analyses below is redundant, we conducted correlation analyses on the response amplitude and the delay-to-peak contrasts for total AG and each subnuclei group. None of the correlations reached statistical significance at a liberal $p<.10$ threshold: (1) total AG $[r=-.29]$, (2) CeM group [ $r=-.14]$; (3) BA group $[r=-.38]$; (4) LA group $[r=-.24]$. This suggests that our amplitude and delay-to-peak data accounted for unique aspects of hemodynamic response.

\section{Imaging Analysis}

Behavioral data analysis was performed on the in-scanner rating task using a repeated-measure ANOVA. This analysis showed a main effect of picture category $[F(2,72)=224.79, p<.001$, 
$\left.\eta^{2}=.90\right]$ such that participants rated the Neu pictures as least emotional $(M=1.16, \mathrm{SE}=0.07)$ and the High Emo pictures as most emotional $(M=3.11, \mathrm{SE}=0.1)$. Holm-Bonferroni-corrected pair-wise comparisons demonstrated that each picture type was significantly different from its adjacent picture type (i.e., Neu $<$ Low Emo $<$ Med Emo $<$ High Emo). However, due to the lack of statistical separation in IAPS-based valence ratings between Med and High Emo pictures (as reported above) and because seven of our participants assigned less than 10 pictures as highly emotional, we collapsed the Med and High Emo picture categories into a single negative emotional category for fMRI analyses. This allowed for an increased number of trials in which participants were likely to have perceived the pictures as being "highly" or "moderately" emotional. Since our research interest was limited to detecting differences in sensitivity of the AG subnuclei groups to negative emotion, we did not analyze BOLD response estimates from the Low Emo category. To ensure that visual complexity of the High/Med Emo stimuli was similar to visual complexity of the Neu stimuli, we compared spatial frequencies of these two stimuli groups using Image Statistics Toolbox for MATLAB (Bainbridge and Oliva, 2015; Torralba and Oliva, 2003). Spatial frequency analysis revealed that visual complexity of the stimuli in the combined High/Med Emo category was equal to visual complexity of the stimuli in the Neu category. SPSS (v. 21; IBM Inc., Armonk, NY) was used to perform random-effects analyses on the response amplitude and the delay-to-peak difference scores between the collapsed High/Med Emo category and Neu category (in the results section called simply negative minus neutral contrast). No correction for sphericity assumption violations was required, as our data did not violate these assumptions in any of the response amplitude or delayto-peak tests. Post-hoc comparisons (6 for response amplitude, and 6 for response latency) employed Holm-Bonferroni correction to control for Type I error inflation. Only family-wise 
error (FWE) corrected $p$-values are reported.

\section{Classification Analysis}

A sequential minimal optimization algorithm, from the MATLAB Statistics Toolbox (The MathWorks Inc., Natick, MA) was used to train binary linear support vector machine (SVM) classifiers on standardized BOLD response amplitude and delay-to-peak parameters from anatomically defined ROIs. A box constraint penalty parameter search was carried out on a base10 logarithmic scale $\left(C=10^{-4.0}, 10^{-3.9}, \ldots, 10^{2.4}, 10^{2.5}\right)$ using an internal 9-fold cross-validation loop to find the optimal penalty parameter without excessively over-fitting the model. Classifier performance was assessed using an external 10-fold cross-validation loop. To minimize classifier variability due to random partitioning of the data into train/test folds, the classifier was trained and assessed 200 times for each ROI. The average classification accuracy was computed for all ROIs and rounded to the nearest integer. Rather than make any assumptions about the null distributions of our classifiers, we chose to empirically estimate these null distributions using permutation tests. During this procedure the stimulus category label ['neutral', 'negative] was randomly shuffled 1500-5000 times (depending on the level of precision demanded by each hypothesis test), and the classification procedure was repeated each time. Subsequently, statistical tests were conducted to test against the null hypothesis that stimulus category labels have no meaning and observed classification accuracy could have resulted from random sampling only. To account for null distribution differences between classifiers trained on data from different ROIs, we report classification accuracies above or below the median of the corresponding null distributions. Only Holm-Bonferroni-corrected $p$-values are reported for classifier performance comparisons (4 tests for ROI comparison; 3 tests for CeM comparison). 


\section{Intra-Amygdala Functional Connectivity}

For intra-AG functional connectivity analysis, EPI timecourses were preprocessed using CONN toolbox (v. 13.o; Whitfield-Gabrieli and Nieto-Castanon, 2012). After correcting for head motion, 5 principle eigenvariates from WM and 5 from CSF voxels were co-varied out of the realigned timecourses. This procedure removes spurious autocorrelations from the data that are a byproduct of physiological noise. Subsequently, we regressed out task-induced signal changes evoked by each stimulus category. To model stimulus-evoked signal changes, we used a twoparameter model of hemodynamic response, represented by optimized double-gamma function (as previously estimated using SIMPLEX algorithm) and its first-order derivative. Next, time points with excessive head motion and signal spikes (as previously identified by ART) were identified and removed from the data. Resulting timecourses were band-pass filtered (Low $=$ $0.09 \mathrm{~Hz}$, High $=0.008 \mathrm{~Hz}$ ). Because serial autocorrelation (i.e. BOLD data in sequential TRs is correlated) violates assumptions of least-squares algorithm, and results in biased correlation coefficients, we carried out a partial autocorrelation analysis to identify the most appropriate autoregressive (AR) model. This analysis revealed that, in most cases, lag 5 was the last lag to produce a significant autocorrelation. Thus, the AR5 model sufficiently corrected for serial autocorrelations and was used for the reported results. For each pairing of the three subnuclei groups we performed partial correlation analysis, controlling for the other ROI (e.g., correlation between the CeM group and the BA group, controlling for the LA nucleus), on the timecourses. These correlation coefficients were then converted to Fisher's Z-scores (Fisher, 1921). Each participant's $Z$-transformed correlation values were averaged across all runs, and were carried over for the random-effects analysis, which was performed in SPSS. Where appropriate, HuynhFeldt correction for sphericity violation was applied. For simplicity of interpretation, group Z- 
score values were inverse-transformed into corresponding correlation coefficients. Post-hoc comparisons of intra-AG connectivity also employed Holm-Bonferroni correction for multiple comparisons (6 tests). Only FWE-corrected findings are reported.

\section{RESULTS}

\section{Total amygdala}

First, we performed a paired-samples t-test to determine whether left and right total AG differed in their sensitivity to negative emotional stimuli. We observed no laterality effects [amplitude: $t(24)=1.45, p=.16$, Cohen's $d=0.29$; delay to peak: $t(24)=1.16, p=.26, d=0.23]$.

Therefore, data for each emotional condition of interest (i.e., negative and neutral) was averaged across hemispheres. To examine differences in the total AG's response to negative versus neutral items, paired-samples $t$-tests were performed on the amplitude and delay-to-peak data.

Consistent with prior findings (Ball et al., 2009; Sergerie et al., 2008), analysis of the amplitude data showed increased sensitivity in bilateral AG to negative stimuli $[t(24)=4.804, p<.0001, d$ $=0.96, M_{\text {diff }}=0.16 \%$ signal change $($ Fig. 5a, 6a). However, analysis of delay to peak showed only a trend with later time to peak in response to negative emotion $[t(24)=1.89, p=.071, d=$ $\left.0.23, M_{\text {diff }}=0.36 \mathrm{~s}\right]($ Fig. 5a, 6b).

\section{Amygdala subnuclei groups}

\section{Amplitude of response}

To determine whether the AG subnuclei groups differentially respond to negative emotional stimuli we calculated difference scores by subtracting amplitude values in response to neutral stimuli from those in response to negative emotional stimuli. The resulting values indicated the degree of emotional sensitivity. A repeated-measures ANOVA with two factors [Hemisphere 
(left, right); Subnuclei Group (CeM, BA, LA)] revealed a significant main effect of Subnuclei Group $\left[F(2,48)=4.40, p=.018, \eta^{2}=.061\right]$, demonstrating that the AG subnuclei groups responded differently to the negative stimuli. Because neither the main effect of hemisphere was significant $\left[F(2,48)=1.63, p=.21, \eta^{2}=.014\right]$, nor the two-way interaction $[F(2,48)=0.90, p=$ $\left..41, \eta^{2}=.014\right]$, we concluded that the effect of the negative emotional stimuli on the AG subnuclei groups was consistent across hemispheres. Therefore, we performed simple effects analyses on the difference scores averaged across the hemispheres.

To determine how the AG subnuclei groups differed in their sensitivity to emotion we first examined the difference scores for each subnuclei group separately. The CeM group responded significantly to the negative emotional stimuli $\left[t(24)=4.09, p=.003, d=0.82, M_{\text {diff }}=0.28 \%\right.$ signal change $\left(\mathbf{F i g}\right.$. 5b, 6a), as did the BA group $\left[t(24)=3.24, p=.017, d=0.64, M_{\text {diff }}=0.14 \%\right.$ signal change] (Fig. 5c, 6a). The LA nucleus, however, did not differentially respond to the emotional stimuli $[t(24)=1.81, p=.17, d=0.36]$ (Fig. 5d, 6a). Comparing the AG subnuclei to each other showed that the CeM group was more sensitive to the negative stimuli than the LA nucleus $\left[t(24)=2.82, p=.038, d=0.56, M_{\text {diff }}=0.21 \%\right.$ signal change $]$. However, neither the CeM group nor the LA nucleus differed significantly from the BA group $[t(24)=1.84, p=.23, d$ $=0.37 ; t(24)=1.02, p=.32, d=0.20$, respectively].

\section{Delay to peak BOLD response}

To determine whether the AG subnuclei groups display latency differences in hemodynamic response due to negative stimulus processing we calculated latency difference scores by subtracting delay-to-peak values in response to neutral stimuli from those in response to negative stimuli. A repeated-measures ANOVA with two factors [Hemisphere (left, right); Subnuclei Group (CeM, BA, LA)] was performed on these difference scores. The main effect of subnuclei 
group showed a trend towards significance $\left[F(2,48)=2.53, p=.090, \eta^{2}=.039\right]$ suggesting that subnuclei might differ in their peak latency in response to emotional relative to neutral stimuli. Neither the main effect of hemisphere $\left[F(1,24)=2.59, p=.12, \eta^{2}=.010\right]$ nor the two-way interaction $\left[F(2,48)=0.06, p=.98, \eta^{2}<.001\right]$ was significant, demonstrating that latency differences between emotional and neutral stimuli were consistent across hemispheres. Consequently, the delay-to-peak data was averaged across the two hemispheres.

To determine which AG subnuclei group, if any, was driving the marginal main effect of the Subnuclei Group in the above analysis we examined the latency difference scores for each AG subnuclei group separately. The CeM group showed significantly later BOLD response peak for the negative emotional stimuli compared to neutral stimuli $[t(24)=3.245, p=.021, d=0.65$, $\left.M_{\text {diff }}=0.83 \mathrm{~s}\right]$ (Fig. 5b, 6b). The LA nucleus and the BA group, however, did not show significant emotion-related differences in BOLD response latency $[t(24)=2.087, p=.19, d=$ $0.42, M_{\text {diff }}=0.46 \mathrm{~s} ; t(24)=1.005, p=.32, d=0.20, M_{\text {diff }}=0.19 \mathrm{~s}$, respectively] (Fig. 5c-d, 6b). Although, the CeM group had the largest difference in BOLD response latency due to negative stimulus processing, this difference was only marginally larger than the difference found in the BA group $\left[t(24)=2.51, p=0.097, d=0.50, M_{\text {diff }}=0.64 \mathrm{~s}\right]$, and no different from the latency difference in the LA nucleus $[t(24)=1.10, p=.84, d=0.22]$. The difference in the delay to peak to emotional relative to neutral stimuli did not differ between the LA nucleus and the BA group $[t(24)=1.04, p=0.61, d=0.21]$. These results suggest that sensitivity to negative emotional stimuli, as measured by the delay-to-peak contrast, is likely different between the AG subnuclei groups.

\section{Prediction of image emotional content based on BOLD response parameters}

To determine whether BOLD response parameters (response amplitude and delay to peak) from 
any of the AG subnuclei groups are better at predicting the emotional content of a stimulus than BOLD response parameters from the total AG we trained 4 linear Support Vector Machine (SVM) classifiers, one for the total AG and one for each AG subnuclei group, on 4 features from each participant [average left/right hemisphere amplitude of response, average left/right hemisphere delay to peak]. The classifier trained on the data from the total AG performed statistically no better than chance [59\% accuracy ( $14 \%$ above median), $p>0.1]$. The classifiers trained on the data from the BA group and the data from the LA nucleus were even less accurate at predicting the emotional content of the stimuli [ $52 \%$ accuracy $(6 \%$ and $8 \%$ above median, respectively), $p>0.1]$. The only classifier that succeeded at predicting the emotional category of a stimulus better than chance was the classifier trained on BOLD response parameters from the CeM group [71\% accuracy (24\% above median), $p<0.01]$. This demonstrated that BOLD measures from the CeM group are better at predicting the emotional content of a stimulus than BOLD measures from the total AG or any other AG subregion (Fig. 7a). To ensure that neither the CeM BOLD response amplitude nor the CeM BOLD response delay to peak was redundant to the overall classification model, we trained two separate CeM classifiers, one only on the amplitude data, and the other only on the delay-to-peak data. While both of these classifiers performed better than chance [Amplitude accuracy $=62 \%(16 \%$ above median $), p<0.05$; Latency accuracy $=70 \%(19 \%$ above median $), p<0.05]$, the CeM model trained on both parameters at the same time performed best (Fig. 7a,b).

\section{Intra-amygdala connectivity}

To examine potential pathways of information flow within the $\mathrm{AG}$, we performed a functional connectivity analysis between the AG subnuclei groups. A repeated-measures ANOVA was performed on Fisher Z-transformed partial correlations between the AG subnuclei groups (called 
edges) with Hemisphere [left, right] and Edge $[\mathrm{CeM} \leftrightarrow \mathrm{BA}, \mathrm{CeM} \leftrightarrow \mathrm{LA}, \mathrm{BA} \leftrightarrow \mathrm{LA}]$ as factors. We observed a significant main effect of Edge $\left[F(2,48)=17.69, p<.00001, \eta^{2}=.235\right]$, demonstrating that three edges representing intra-AG connectivity differed in the strength of their correlation. The main effect of hemisphere was not significant $[F(1,24)=3.38, p=.079$, $\left.\eta^{2}=.012\right]$, and neither was the two way interaction $\left[F(2,48)=0.535, p=.54, \eta^{2}=.008\right]$, demonstrating that the pattern of intra-AG connectivity was consistent across hemispheres.

Based on partial correlation analysis, statistically significant connectivity existed between all of the AG subnuclei groups (all $p$ 's $<0.01$ ), suggesting that all subnuclei are functionally related to each other (Fig. 8). Furthermore, all partial correlations statistically differed from each other (all $p ' s<0.05)$ : $\mathrm{BA} \leftrightarrow \mathrm{LA}$ connectivity was the strongest, followed by $\mathrm{CeM} \leftrightarrow \mathrm{BA}$, while $\mathrm{CeM} \leftrightarrow$ LA connectivity was the weakest (Fig. 8).

\section{Discussion}

Using high-resolution fMRI we demonstrated for the first time that anatomically-defined subnuclei groups of the human AG respond differently to negative emotional stimuli, and that strength of functional connectivity between various AG subregions is not identical. Although most prior functional neuroimaging studies analyzed the AG as a single homogeneous structure, our findings provide strong evidence in support of functional specialization within the human AG. As measured by BOLD response amplitude, the CeM group was most sensitive to the negative emotional stimuli, followed by the BA group, while the LA nucleus was largely insensitive to negative emotions. The CeM AG also demonstrated clear latency differences in its hemodynamic response to the negative emotional stimuli, while the BA group and the LA nucleus did not. Critically, only the activity in the CeM group was able to predict the emotional 
content of the stimuli based on BOLD response parameters in a classification analysis.

Examination of the intra-AG functional connectivity demonstrated that fMRI signal covariance was the strongest between the BA group and the LA nucleus, intermediate between the BA group and the CeM group, and the weakest between the LA nucleus and the CeM group.

Although, some previous studies attempted to elucidate functions of the AG subnuclei in negative stimulus processing, they were substantially limited by spatial resolution (e.g. Styalidis et al., 2014; Yoder et al., 2015). This limitation is most pronounced for the nuclei of the dorsal AG. Histological experiments revealed that the CeM AG is smaller than $150 \mathrm{~mm}^{3}$ (Brabec et al., 2010; Garcia-Amado and Prensa, 2012). Consequently, the CeM AG is only 2-9 voxels large on fMRI images with 16-64 $\mathrm{mm}^{3}$ voxels. Here, we optimized all our acquisitions to guarantee sufficient spatial resolution to study the effects of negative stimulus processing not only on the AG subnuclei groups from the ventral AG, but also from the dorsal AG.

The overall efficacy of our experimental paradigm at eliciting AG activation and our analysis approach at detecting it was validated by our whole AG results, which demonstrated increased sensitivity to negative emotional stimuli in the bilateral AG. These results are in agreement with the extant fMRI literature (Sergerie et al., 2008), case studies of patients with the AG lesions (Adolphs, et al., 1994, 1995; Broks et al., 1998), and non-human fear conditioning experiments (Davis, 1992; Kapp et al., 1994), providing further support to the body of literature on AG's involvement in processing of high arousing negative emotions. In agreement with similar fMRI studies as reported in a large meta-analysis of fMRI literature on the human AG by Sergerie et al. (2008), we observed no laterality effects in any measure of hemodynamic response in the total AG or any of its subnuclei. 


\section{Differential engagement of the amygdala subnuclei groups in response to negative emotional}

stimuli

The main finding from the current study showed preferential sensitivity of the CeM AG to highly arousing negative emotional stimuli. The $\mathrm{CeM}$ group was sensitive to negative emotion in all of the analyses we performed. Its BOLD response to negative pictures was greater and peaked later, when compared to BOLD response to neutral pictures.

While we did not initially have any predictions about differences in delay-to-peak latency as a function of emotion, there are several lines of evidence that may help explain this finding. It is reasonable to expect that non-task-related differences across ROIs in the timing of the BOLD response may be due to vasculature, neuronal, or neurovascular coupling effects that are similar for one area of tissue but not another (Kim and Ogawa, 2012; Logothetis and Wandell, 2004). However, task-related differences in BOLD latency within the same ROI are likely reflective of differences in the underlying neuronal activity (Formisano and Goebel, 2003; Pernet et al., 2004). In this regard, the delay to peak for emotional relative to neutral stimuli in the CeM AG is likely due to emotion-specific changes in neuronal activity. Animal work has shown that increasing stimulation frequency of somatosensory neurons produces higher and later hemodynamic response peaks (Martin et al., 2006). Similarly, our CeM amplitude and latency findings might simply represent greater stimulation of the $\mathrm{CeM}$ neurons as a consequence of the negative stimulus processing. It is also possible that emotion-related firing rates within the human AG are of different durations. Consequently, our CeM latency findings might represent longer lasting activation of the CeM group driven by negative stimulus processing. Although we did not directly test differences in the duration (i.e., dispersion) of the BOLD response, this explanation is supported by recent animal literature which demonstrated subnuclei-specific 
variations in neuronal firing durations during fear conditioning experiments (Duvarci and Paré, 2014). Additionally, the motivational processes evoked by the AG subnuclei may also be of relevance, e.g., if the AG activity corresponds to not only a response due to negative emotion, but also approach- vs. avoidance-related processes, which involve interactions with the prefrontal cortex and insula (Cardinal et al., 2002; Harmon-Jones et al., 2013; Madan, 2013). As our study was not designed to assess emotion-driven causes of hemodynamic latency differences, future research is needed determine the exact cause of these findings.

Previous high-resolution fMRI studies (Table 1) reported involvement of corticomedial and CeM AG in various aspects of Pavlovian conditioning and instrumental processes (Boll et al., 2013; Prévost et al., 2011, 2012, 2013). However, because prior high-resolution fMRI literature did not report CeM activations in response to aversive visual stimuli, we must turn to animal experiments to gain greater insight into biological significance of our results. Non-human fearconditioning experiments have shown that the Ce nucleus of the AG is the primary regulator of behavioral and autonomic responses. Through its connections with the lateral and paraventricular hypothalamus, Ce AG modulates heart rate, blood pressure, corticosteroid release, and skin conductance (Davis and Whalen, 2001). Ce projections to the ventral tegmental area, locus coeruleus, and basal forebrain modulate arousal, vigilance, and attention, while its projections to the periaqueductal grey and cranial nerve nuclei control freezing and escape behaviours (Davis and Whalen, 2001). Recent primate work on functional role of the Ce AG arrived at similar conclusions (Kalin et al., 2004).

Similarly, images of negative valence and high arousal have been found to elicit increased skin conductance response, decreased heart rate, and increased attention in humans (Lang, et al. 1998). Therefore, based on extant knowledge of processes underlying fear conditioning, and 
commonalities between different organisms' physiological responses to unpleasant stimuli, we think that the CeM activation reported in the current study, is related to its involvement in regulation of autonomic, endocrine, and behavioural responses induced by negative stimulus processing.

Our parametric response amplitude results also demonstrated some sensitivity of the BA group nuclei to negative emotion. Animal work has shown that the BA nucleus receives most of its inputs from two sources, the LA nucleus of the AG, and the orbitofrontal cortex, while its outputs mainly project to the Ce AG and the striatum, where they provide context-dependent modulation of emotional processing (Freese and Amaral, 2009), and contribute to regulation of instrumental behaviors (LeDoux and Schiller, 2009).

\section{Intra-amygdala connectivity}

In general, our connectivity results are in agreement with previous animal work. In rodents the LA nucleus acts as the sensory interface of the AG, and receives inputs from primary sensory systems (Phelps and LeDoux, 2005). However, activation of Ce output neurons is necessary for physiological and behavioural changes that increase chances of effectively coping with a biologically relevant stimulus. Although, direct inputs into the CeM group from the LA nucleus exist (Pitkänen et al., 1997), those are few in comparison to the indirect pathway through the B and AB nuclei (Duvarci and Paré, 2014; Freese and Amaral, 2009). Similarly, our results showed the strongest fMRI signal covariance was between the BA group and the LA nucleus, while the weakest communication was between the LA nucleus and the CeM group.

Even though our results are in agreement with animal literature, they are not consistent with another human functional connectivity study (Roy et al., 2009), which reported negative 
correlation patterns within the right AG, and observed no connectivity between the subnuclei of the left AG. These differences in connectivity measurements may have occurred because Roy et al. (2009) relied on Amunts et al. (2005) probabilistic atlas to define the AG subnuclei ROIs in MNI space, while we used Mai (2008) atlas to manually segment the AG ROIs in native space. Although, our connectivity findings were very robust and reliable, they should still be interpreted with caution because our correlation coefficients are fairly small. It is currently unknown whether such low correlation coefficients are a byproduct of lower SNR produced by highresolution acquisition or whether they represent weak intra-AG communication. Nonetheless, our findings conclusively demonstrated that the AG subnuclei groups interact differently with each other.

\section{Hemodynamic properties of the amygdala subnuclei groups}

The current study demonstrated that time and amplitude domains of BOLD response are not necessarily correlated and can provide unique information about the hemodynamic response of a region. Many prior fMRI studies of the AG relied on the canonical hemodynamic response function to model BOLD response (e.g. Ball et al., 2007; Boll et al, 2011; Davis et al., 2010; Gamer et al. 2010; Frühholz and Grandjean, 2012; Prévost et al. 2011). When constructing activation contrasts, this approach assumes that BOLD response across various conditions varies only in its amplitude. Friston et al. (1998) proposed the use of the temporal derivative to account for the delay-induced variations in hemodynamic response. Later, Calhoun et al. (2004) demonstrated that incorporating the derivative and non-derivative terms into response amplitude contrasts produces more accurate estimates of BOLD amplitude differences between various conditions. Furthermore, as was demonstrated by Henson et al. (2002), BOLD response timing effects may be condition-dependent, and can provide additional information. In our results, we 
demonstrated that hemodynamic response amplitude and latency parameters are, at times, independent of each other. Our parametric results showed that for some ROIs, there was difference in BOLD response amplitude between the two conditions (BA group), while other ROIs displayed differences in time and amplitude domains (CeM group). We also showed that multivariate methods might benefit from including both of these BOLD response parameters when training a model. For instance, our best CeM classification model was trained on both response amplitude and delay to peak.

The current study also demonstrated that employing univariate hypothesis testing in conjunction with multivariate classification provides additional insight into properties of the fMRI data. In our univariate findings, both the CeM and the BA group were sensitive to negative emotion. However, when we used the same BOLD response parameters in a multivariate classification analysis, we discovered that only BOLD response from the CeM group could predict above chance which stimulus category these parameters represent. This suggests that while mean responsiveness to negative stimuli was higher in both subnuclei groups, BOLD response parameters for neutral and negative stimuli were clearly separable in the CeM group, but not in the BA group, suggesting that the nuclei of the CeM group process those stimuli differently than the nuclei of the BA group.

\section{Limitations and future directions}

In the current study we relied on ROI analysis to study the AG subnuclei. While ROI techniques provide great anatomical accuracy and precision, they are largely insensitive to dispersed activation patterns. This important caveat likely accounts for the seeming discrepancy between the vital role that the LA nucleus plays in non-human conditioning experiments (Phelps and 
LeDoux, 2005) and our fMRI-based LA activation findings. There is evidence in animal literature that only $20 \%$ of cells in the LA nucleus show plasticity-dependent modulation of sensitivity in response to threat (Duvarci and Paré, 2014). If there are distributed emotionsensitive neural populations in the human LA nucleus, future studies employing pattern recognition techniques might be able to identify them. Another caveat of our analysis procedure is reliance on mathematical interpolation to measure BOLD response timing. Our raw fMRI data was acquired with a 2-second TR, but we report sub-second differences in BOLD response delay to peak. Although future studies employing multi-band sequences with shorter TR are required to verify our latency findings, we do not consider our mathematical interpolation to be a major concern because we relied on previously researched properties of the hemodynamic response in our parameter estimation procedures (Devonshire et al., 2012; Kim and Ogawa, 2012; Logothetis et al., 2001; Logothetis and Wandell, 2004; Martin et al., 2006; Pernet, 2014).

Furthermore, future fMRI studies of the AG subnuclei need to investigate the effects of emotional valence and gender. Although there is substantial evidence that positive emotions engage the AG (Ball et al., 2009; Sergerie et al., 2008), it is currently unclear how the AG subnuclei investigated here would respond to positive versus negative emotional stimuli. The need to investigate the responsiveness of the AG subnuclei to positive stimuli is supported by recent animal work, which suggests that positive and negative stimuli are processed by different pathways within the AG (Namburi et al., 2015). There is also some debate concerning the influence of gender on the AG function (Sergerie et al., 2008). Even though this study was not designed to address this question, in preliminary analyses, we included gender as a between subjects factor, but did not observe a main effect of gender, nor any interactions including it (all $p$ 's $>.1)$. 


\section{Conclusions}

In the current study, we used anatomical landmarks to extract BOLD signal from distinct AG subnuclei groups, and directly compared their activations in response to negative emotional stimuli. Our results showed that the CeM AG is particularly responsive to negative emotions. We also demonstrated that human intra-AG functional connectivity is consistent with animal literature. Future high-field high-resolution fMRI studies of emotional processing will allow researchers to further understand the structure-function relationship of the human AG and its subnuclei, as well as the roles that the AG subnuclei play in neuropsychiatric disorders.

\section{Author Contributions}

Conceptualization, S.H., A.T.S., and N.V.M.; Data acquisition, S.H., F.O., P.S., and N.V.M., fMRI sequence development, C.A.B., and C.B.; Data analysis, S.H., A.A.S., C.R.M., A.T.S., and N.V.M.; Manuscript writing, S.H., A.A.S., C.R.M., A.T.S., and N.V.M. All co-authors reviewed and approved the final version of the manuscript.

\section{Acknowledgements}

We thank Matt R. Brown, Tyler M. Rolheiser, and Alexander Lebedev for their suggestions in improving our data analysis methodology. This project was supported by the Canadian Institutes of Health Research (CIHR) operating grant to N.V.M. S.H. was supported by Sir Fredrick Banting and Dr. Charles Best CIHR Doctoral scholarship. C.R.M. was supported by the National Science and Engineering Research Council (NSERC) Doctoral Scholarship. C.A.B. was supported by NSERC Vanier Doctoral scholarship. C.B. was supported by the Alberta Innovates Health Solutions (AIHS) Scientist Award. 


\section{References}

Adolphs, R., Gosselin, F., Buchanan, T.W., Tranel, D., Schyns, P., and Damasio, A.R. (2005). A mechanism for impaired fear recognition after amygdala damage. Nature, 433, 68-72

Adolphs, R., Tranel, D., Damasio, H., and Damasio, A.R. (1994). Impaired recognition of emotion in facial expressions following bilateral damage to the human amygdala. Nature, $372,669-672$.

Adolphs, R., Tranel, D., Damasio, H., and Damasio A.R. (1995). Fear and the human amygdala. J. Neurosci., 15, 5879-5891.

Amunts, K., Kedo, O., Kindler, M., Pieperhoff, P., Mohlberg, H., Shah, N.J., Habel, U., Schneider, F., and Zilles, K. (2005). Cytoarchitectonic mapping of the human amygdala, hippocampal region and entorhinal cortex: intersubject variability and probability maps. Anat. Embryol., 210, 343-352.

Bach, D.R., Weiskopf, N., and Dolan, R.J. (2011). A stable sparse fear memory trace in human amygdala. J. Neurosci., 31, 9383-9389.

Bainbridge, W.A., and Oliva, A. (2015). Interaction envelope: Local spatial representations of objects at all scales in scene-selective regions. NeuroImage, doi:10.1016/j.neuroimage.2015.07.066.

Ball, T., Derix, J., Wentlandt, J., Wieckhorst, B., Speck, O., Schulze-Bonhage, A., and Mutschler, I. (2009). Anatomical specificity of functional amygdala imaging of responses to stimuli with positive and negative emotional valence. J. Neurosci. Methods, 180, 57-70.

Ball, T., Rahm, B., Eickhoff, S.B., Schulze-Bonhage, A., Speck, O., and Mutschler, I. (2007). Response properties of human amygdala subregions: evidence based on functional MRI combined with probabilistic anatomical maps. PLoS ONE, 2, e307. 
Balleine, B.W., and Killcross, S. (2006). Parallel incentive processing: an integrated view of amygdala function. Trends Neurosci., 29, 272-279.

Barret, L.F. (2005). Valence is a basic building block of emotional life. J. Res. Pers., 40, 35-55.

Boll, S., Gamer, M., Gluth, S., Finsterbusch, J., and Büchel, C. (2013). Separate amygdala subregions signal surprise and predictiveness during associative fear learning in humans. Eur. J. Neurosci., 37, 758-767.

Boll, S., Gamer, M., Kalisch, R., and Büchel, C. (2011). Processing of facial expressions and their significance for the observer in subregions of the human amygdala. NeuroImage, 56, 299-306.

Boubela, R.N., Kalcher, K., Huf, W., Seidel, E.M., Derntl, B., Pezawas, L., Našel, C., and Moser, E. (2015). fMRI measurements of amygdala activation are confounded by stimulus correlated signal fluctuation in nearby veins draining distant brain regions. Sci. Rep., 5 , 10499.

Brabec, J., Rulseh, A., Hoyt, B., Vizek, M., Horinek, D., Hort, J., and Petrovicky, P. (2010). Volumetry of the amygdala - an anatomical study. Psychiatry Res., 182, 67-72.

Bradley, M.M., and Lang, P.J. (1994). Measuring emotion: the self-assessment manikin and the semantic differential. J. Behav. Ther. Exp. Psychiatry, 25, 49-59.

Broks, P., Young, A.W., Maratos, E.J., Coffey, P.J., Calder, A.J., Isaac, C.L., Mayes, A.R., Hodges, J.R., Montaldi, D., Cezayirli, E., Roberts, N., and Hadley, D. (1998). Face processing impairments after encephalitis: amygdala damage and recognition of fear. Neuropsychologia, 36, 59-70.

Brown, T.A., Di Nardo, P.A., Lehman, C.L., and Campbell, L.A. (2001). Reliability of DSMIV anxiety and mood disorders: implications for the classification of emotional disorders. J. of Abn. Psychol, 110, 49-58.

Büchel, C., Morris, J., Dolan, R.J., and Friston, K.J. (1998). Brain systems mediating aversive conditioning: an event-related fMRI study. Neuron, 20, 947-957.

Calhoun, V.D., Stevens, M.C., Pearlson, G.D., and Kiehl, K.A. (2004). fMRI analysis with the general linear model: removal of latency-induced amplitude bias by incorporation of hemodynamic derivative terms. NeuroImage, 22, 252-257.

Cardinal, R.N., Parkinson, J.A., Hall, J., and Everitt, B.J. (2002). Emotion and motivation: the role of the amygdala, ventral striatum, and prefrontal cortex. Neurosci. Biobehav. Rev., 26, 321-352. 
Davis, M. (1992). The role of the amygdala in conditioned fear. In The Amygdala:

Neurobiological Aspects of Emotion, Memory, and Mental Dysfunction, J. Aggleton, ed. (New York, NY: Wiley), pp. 255-305.

Davis, C.F., Johnstone, T., Mazzulla, E.C., Oler, J.A., and Whalen, P.J. (2010). Regional response differences across the human amygdaloid complex during social conditioning. Cereb. Cortex, 20, 612-621.

Davis, M., and Whalen, P.J. (2001). The amygdala: vigilance and emotion. Mol. Psychiatry, 6, $13-34$.

Devonshire, I.M., Papadakis, N.G., Port, M., Berwick, J., Kennerly, A.J., Mayhew, J.E.W., and Overton, P.G. (2012). Neurovascular coupling is brain region-dependent. NeuroImage, 59, 1997-2006.

Di Nardo, P.A., Brown, T.A., and Barlow, D.H. (1994). Anxiety Disorders Interview Schedule for DSM-IV - Lifetime version (ADIS-IV-L), Psychological Corporation, San Antonio, TX.

Dolcos, F., LaBar, K.S., and Cabeza, R. (2004). Interaction between the amygdala and the medial temporal lobe memory system predicts better memory for emotional events. Neuron, 42, 855-863.

Duvarci, S., and Paré, D. (2014). Amygdala microcircuits controlling learned fear. Neuron, 82, 966-980.

Entis, J.J., Doerga, P., Barrett, L.F., and Dickerson, B.C. (2012). A reliable protocol for the manual segmentation of the human amygdala and its subregions using ultra-high resolution MRI. NeuroImage, 60, 1226-1235.

Fisher, R.A. (1921). On the 'probable error' of a coefficient of correlation deduced from a small sample. Metron, 1, 3-32.

Formisano, E., and Goebel, R. (2003). Tracking cognitive processes with functional MRI mental chronometry. Curr. Opin. Neurobiol., 13, 174-181.

Friston, K.J., Fletcher, P., Josephs, O., Holmes, A., Rugg, M.D., and Turner, R. (1998). Eventrelated fMRI: characterizing differential responses. NeuroImage, 7, 30-40.

Freese, J.L., and Amaral, D.G. (2009). Neuroanatomy of the primate amygdala. In The Human Amygdala, P.J. Whalen, and E.A. Phelps, eds. (New York, NY: Guilford Press), pp. 3-42.

Frühholz, S., and Grandjean, D. (2013). Amygdala subregions differentially respond and rapidly adapt to threatening voices. Cortex, 49, 1394-1403. 
Gamer, M., Zurowski, B., and Büchel, C. (2010). Different amygdala subregions mediate valence-related and attentional effects of oxytocin in humans. Proc. Natl. Acad. Sci. U. S. A., 107, 9400-9405.

Garcia-Amado, M., and Prensa, L. (2012). Stereological analysis of neuron, glial, and endothelial cell numbers in the human amygdaloid complex. PLoS One, 7: e38692.

Gottfried, J.A., O’Doherty, J., and Dolan, R.J. (2002). Appetitive and aversive olfactory learning in humans studied using event-related functional magnetic resonance imaging. J.

Neurosci., 22, 10829-10837.

Grant, M.M., Wood, K., Sreenivasan, K., Wheelock, M., White, D., Thomas, J., Knight, D.C., and Deshpande, G. (2015). Influence of early life stress on intra- and extra-amygdaloid causal connectivity. Neuropsychopharmacology, 40, 1782-1793.

Griswold, M.A., Jakob, P.M., Heidemann, R.M., Nittka, M., Jellus, V., Wang, J., Kiefer B., and Haase, A. (2002). Generalized autocalibrating partially parallel acquisitions (GRAPPA). Magn. Reson. Med., 47, 1202-1210.

Handwerker, D.A., Ollinger, J.M., and D’Esposito, M. (2004). Variation of BOLD hemodynamic responses across subjects and brain regions and their effects on statistical analyses. NeuroImage, 21, 1639-1651.

Harmon-Jones, E., Gable, P.A., and Price, T.F. (2013). Does negative affect always narrow and positive affect always broaden the mind? Considering the influence of motivational intensity on cognitive scope. Curr. Dir. Psychol. Sci., 22, 301-307.

Henson, R.N.A., Price, C.J., Rugg, M.D., Turner, R., and Friston, K.J. (2002). Detecting latency differences in event-related BOLD responses: application to words versus nonwords and initial versus repeated face presentations. NeuroImage, 15, 83-97.

Janak, P.H., and Tye, K.M. (2015). From circuits to behaviour in the amygdala. Nature, 517, 284-292.

Johnston, J.B. (1923). Further contributions to the study of the evolution of the fore-brain. J. Comp. Neurol., 35, 271-482.

Kalin, N.H., Shelton, S.E., and Davidson, R.J. (2004). The role of the central nucleus of the amygdala in mediating fear and anxiety in the primate. J. Neurosci., 24, 5506-5515.

Kapp, B.S., Supple, W.F., and Whalen, P.J. (1994). Effects of electrical stimulation of the amygdaloid central nucleus on neocortical arousal in the rabbit. Behav. Neurosci., 108, 8193.

Kensinger, E.A. (2009). Remembering the Details: Effects of Emotion. Emot. Rev., 1, 99-113. 
Kim, S.G., and Ogawa, S. (2012). Biophysical and physiological origins of blood oxygenation level-dependent fMRI signals. J. Cereb. Blood Flow Metab., 32, 1188-1206.

Kim, H., Somerville, L.H., Johnstone, T., Alexander, A.L., and Whalen, P.J. (2003). Inverse amygdala and medial prefrontal cortex responses to surprised faces. Neuroreport, 14, 23172322.

Kim, H., Somerville, L.H., Johnstone, T., Polis, S., Alexander, A.L., Shin, L.M., and Whalen, P.J. (2004). Contextual modulation of amygdala responsivity to surprised faces. J. Cogn. Neurosci., 16, 1730-1745.

Klumpers F., Morgan, B., Terburg, D., Stein, D.J., and van Honk, J. (2014). Impaired acquisition of classically conditioned fear-potentiated startle reflexes in humans with focal bilateral basolateral amygdala damage. Soc. Cogn. Affect. Neurosci., 10, 1161-1168.

LaBar, K.S., Gatenby, J.C., Gore, J.C., LeDoux, J.E., and Phelps, E.A. (1998). Human amygdala activation during conditioned fear acquisition and extinction: a mixed-trial fMRI study. Neuron, 20, 937-945.

Lang, P.J., Bradley, M.M., and Cuthbert, B.N. (1998). Emotion, motivation, and anxiety: brain mechanisms and psychophysiology. Biol. Psychiatry, 44, 1248-1263.

Lang, P.J., Bradley, M.M., and Cuthbert, B.N. (2008). International Affective Picture System (IAPS): Affective Ratings of Pictures and Instruction Manual. Technical Report A-8. Gainsville, FL: Center for the Study of Emotion and Attention at the University of Florida.

LeDoux, J.E. (2000). Emotion circuits in the brain. Annu. Rev. Neurosci., 23, 155-184.

LeDoux, J.E. (2012). Rethinking the emotional brain. Neuron, 73, 653-676.

LeDoux, J.E. (2014). Coming to terms with fear. Proc. Natl. Acad. Sci. U. S. A., 111, 28712878 .

LeDoux, J.E., and Schiller, D. (2009). The human amygdala: Insights from other animals. In The Human Amygdala, P.J. Whalen, and E.A. Phelps, eds. (New York, NY: Guilford Press), pp. 43-60.

Logothetis, N. K., Pauls, J., Augath, M., Trinath, T., and Oeltermann, A. (2001).

Neurophysiological investigation of the basis of the fMRI signal. Nature, 412, 150-157.

Logothetis, N.K, and Wandell, B.A. (2004). Interpreting the BOLD signal. Annu. Rev. Physiol., $66,735-769$.

Madan, C.R. (2013). Toward a common theory for learning from reward, affect, and motivation: the SIMON framework. Fron. Syst. Neurosci., 7, 59.

Mai, J.K. (2008). Atlas of the Human Brain: Third Edition (New York, NY: Academic Press). 
Malykhin, N.V., Bouchard, T.P., Ogilvie, C.J., Coupland, N.J., Seres, P., and Camicioli, R. (2007). Three-dimensional volumetric analysis and reconstruction of amygdala and hippocampal head, body and tail. Psychiatry Res., 155, 155-165.

Martin, C., Martindale, J., Berwick, J., and Mayhew, J. (2006). Investigating neuralhemodynamic coupling and the hemodynamic response function in the awake rat. NeuroImage, 32, 33-48.

Morris, J.S., Büchel, C., and Dolan, R.J. (2001). Parallel neural responses in amygdala subregions and sensory cortex during implicit fear conditioning. NeuroImage, 13, 10441052.

Morris, J.S., deBois, M., and Dolan, R.J. (2002). Human amygdala responses to fearful eyes. NeuroImage, 17, 214-222.

Murty, V.P., Ritchey, M., Adcock, R.A., and LaBar, K.S. (2010). fMRI studies of successful emotional memory encoding: A quantitative meta-analysis. Neuropsychologia, 48, 34593469 .

Namburi, P., Beyeler, A., Yorozu, S., Calhoon, G.G., Halbert, S.A., Wichmann, R., Holden, S.S., Mertens, K.L., Felix-Ortiz, A.C., Wickersham, I.R., Gray, J.M., and Tye, K.M. (2015). A circuit mechanism for differentiating positive and negative associations. Nature, 520, 675678.

Nelder, J.A. and Mead, R. (1965). A simplex method for function minimization. Comput. J., 7, 308-313.

Pernet, C.R. (2014). Misconceptions in the use of the General Linear Model applied to functional MRI: a tutorial for junior neuro-imagers. Front. Neurosci., 8:1.

Pernet, C., Franceries, X., Basan, S., Cassol, E., Démonet, J.F., and Celsis, P. (2004). Anatomy and time course of discrimination and categorization process in vision: an fMRI study. NeuroImage, 22, 1563-1577.

Phelps, E.A., Delgado, M.R., Nearing, K.I., and LeDoux, J.E. (2004). Extinction learning in humans: the role of the amygdala and vmPFC. Neuron, 43, 897-905.

Phelps, E.A., and LeDoux, J.E. (2005). Contributions of the amygdala to emotion processing: from animal models to human behavior. Neuron, 48, 175-187.

Pitkänen, A., Pikkarainen, M., Nurminen, N., and Ylinen, A. (2000). Reciprocal connections between the amygdala and the hippocampal formation, perirhinal cortex, and postrhinal cortex in rat. A review. Ann. N. Y. Acad. Sci., 911, 369-391. 
Pitkänen, A., Savander, V., and LeDoux, J.E. (1997). Organization of intra-amygdaloid circuitries in the rat: an emerging framework for understanding functions of the amygdala. Trends Neurosci., 20, 517-523.

Prévost, C., Liljeholm, M., Tyszka, J.M., and O’Doherty, J.P. (2012). Neural correlates of specific and general Pavlovian-to-instrumental transfer within human amygdalar subregions: a high-resolution fMRI study. J. Neurosci., 32, 8383-8390.

Prévost, C., McCabe, J.A., Jessup, R.K., Bossaerts, P., and O’Doherty, J.P. (2011). Differentiable contributions of human amygdalar subregions in the computations underlying reward and avoidance learning. Eur. J. Neurosci., 34, 134-145.

Prévost, C., McNamee, D., Jessup, R.K., Bossaerts, P., and O’Doherty, J.P. (2013). Evidence for model-based computations in the human amygdala during Pavlovian conditioning. PLoS Comput. Biol., 9: e1002918.

Roy, K.A., Shehzad, Z., Margulies, D.S., Clare Kelly, A.M., Uddin, L.Q., Gotimer, K., Biswal, B.B., Castellanos, F.X., and Milham, M.P. (2009). Functional connectivity of the human amygdala using resting state fMRI. NeuroImage, 45, 614-626.

Russell, J.A. (1980). A circumplex model of affect. J. Pers. Soc. Psychol., 39, 1161-1178.

Russell, J.A. (2003). Core affect and the psychological construction of emotion. Psychol. Rev., $110,145-172$.

Sah, P., Faber E.S., Lopez de Armentia, M., and Power J. (2003). The amygdaloid complex: anatomy and physiology. Physiol. Rev., 83, 803-834.

Sergerie, K., Chochol, C., and Armony, J.L. (2008). The role of the amygdala in emotional processing: a quantitative meta-analysis of functional neuroimaging studies. Neurosci. Biobehav. Rev., 32, 811-830.

Singhal, A., Shafer, A.T., Russell, M., Gibson, B., Wang, L., Vohra, S., and Dolcos F. (2012). Electrophysiological correlates of fearful and sad distraction on target processing in adolescents with attention deficit-hyperactivity symptoms and affective disorders. Front. Integr. Neurosci., 6:119.

Shafer, A.T., and Dolcos, F. (2012). Neural correlates of opposing effects of emotional distraction on perception and episodic memory: an event-related fMRI investigation. Front. Integr. Neurosci., 6:70.

Shafer, A.T., Matveychuck, D., Penney, T., O’Hare, A.J., Stokes, J., and Dolcols, F. (2012). Processing of emotional distraction is both automatic and modulated by attention: evidence from an event-related fMRI investigation. J. Cogn. Neurosci., 24, 1233-1252. 
Styalidis, C., Ioannides, A.A., Bamidis, P.D., and Papadelis, C. (2014). Amygdala responses to valence and its interaction by arousal revealed by MEG. Int. J. Psychophysiol., 93, 121133.

Terburg, D., Morgan, B.E., Montoya, E.R., Hooge, I.T., Thornton, H.B., Hariri, A.R., Panksepp, J., Stein, D.J., and van Honk, J. (2012). Hypervigilance for fear after basolateral amygdala damage in humans. Transl. Psychiatry, 2, e115.

Torralba, A., and Oliva, A. (2003). Statistics of natural image categories. Network, 14, 391-412.

Wang, L., LaBar, K.S., Smoski, M., Rosenthal, M.Z., Dolcos, F., Lynch, T.R., Krishnan, R.R., and McCarthy G. (2008). Prefrontal mechanisms for executive control over emotional distraction are altered in major depression. Psychiatry Res., 163, 143-155.

Wang, L., McCarthy, G., Song, A.W., and LaBar, K.S. (2005). Amygdala activation to sad pictures during high-field (4 tesla) functional magnetic resonance imaging. Emotion, 5, 1222.

Weiskrantz, L. (1956). Behavioral changes associated with ablation of the amygdaloid complex in monkeys. J. Comp. Physiol. Psychol., 49, 381-391.

Whalen, P.J., Davis, F.C., Oler, J.A., Kim, H., Kim, M.J., and Neta, M. (2009). Human amygdala responses to facial expressions of emotion. In The Human Amygdala, P.J. Whalen, and E.A. Phelps, eds. (New York, NY: Guilford Press), pp. 265-288.

Whalen, P.J., Rauch, S.L., Etcoff, N.L., McInerney, S.C., Lee, M.B., and Jenike, M.A. (1998). Masked presentations of emotional facial expressions modulate amygdala activity without explicit knowledge. J. Neurosci., 18, 411-418.

Whalen, P.J., Shin, L.M., McInerney, S.C., Fischer, H., Wright, C.I., and Rauch, S.L. (2001). A functional MRI study of human amygdala responses to facial expressions of fear versus anger. Emotion, 1, 70-83.

Whitfield-Gabrieli, S. and Nieto-Castanon, A. (2012). Conn: a functional connectivity toolbox for correlated and anticorrelated brain networks. Brain Connect., 2, 125-141.

Yassa, M.A., and Stark, C.E. (2009). A quantitative evaluation of cross-participant registration techniques for MRI studies of the medial temporal lobe. NeuroImage, 44, 319-327.

Yoder, K.J., Porges, E.C., and Decety, J. (2015). Amygdala subnuclei connectivity in response to violence reveals unique influences of individual differences in psychopathic traits in a nonforensic sample. Hum. Brain Mapp., 36, 1417-1428.

Yushkevich, P.A., Piven, J., Hazlett, H.C., Smith, R.G., Ho, S., Gee, J.C., and Gerig, G. (2006). User-guided 3D active contour segmentation of anatomical structures: Significantly improved efficiency and reliability. NeuroImage, 31, 1116-1128. 
Zald, D.H., and Pardo, J.V. (2002). The neural correlates of aversive auditory stimulation. NeuroImage, 16, 746-753.

\section{Figure and Table Captions}

Table 1. Recent high-resolution fMRI studies of the amygdala subnuclei (AG, amygdala; CeM, centromedial group; BLA, basolateral group; LB, laterobasal group).

Figure 1. Brain tissue covered by high-resolution anatomical and high-resolution functional scans. Single participant's full-brain T1-weighted anatomical scan is shown in the background. Violet overlay represents coverage of ultra-high-resolution anatomical 2D FSE scan, which was used for manual amygdala segmentation. Red overlay represents areas captured by highresolution fMRI EPI acquisition. Segmented amygdala is shown in green.

Figure 2. Subdivision of the amygdala on high-resolution inverted T2-weighted structural FSE image. (a). Saggital view of the amygdala with references to coronal slices. (b-f). Coronal views of the amygdala subnuclei groups. Landmarks used to delineate the subnuclei borders are shown as dashed lines and points A and B (see text for details). 
Figure 3. Three-dimensional reconstruction of the amygdala subnuclei groups from a healthy volunteer. (a). lateral view; (b). anterior view; (c). medial view; (d). superior view; (e). posterior view; (f). inferior view. Abbreviations: CeM, centromedial group; BA, basal group; LA, lateral nucleus.

Figure 4. BOLD response in bilateral amygdala, averaged across participants and stimulus categories is shown in blue. A double-gamma function that was optimized to fit the amygdala BOLD response is shown in red (a.u., arbitrary units).

Figure 5. Reconstructed hemodynamic response function for negative (red) and neutral (blue) stimuli in the total amygdala and its subnuclei groups, averaged across hemispheres. Shaded areas represent the standard error of the mean, corrected for inter-individual differences. BOLD response amplitude and delay to peak are shown in brackets for each stimulus category.

Figure 6. Amplitude (a) and Latency (b) negative minus neutral contrasts for the total amygdala and each of its subnuclei groups (CeM, centromedial group; BA, basal group; LA, lateral nucleus). ${ }^{*}$ FWE $p<0.05 ; * *$ FWE $p<0.01$.

Figure 7. (a) Classification accuracy above median of linear SVM classifiers trained on response amplitude and delay-to-peak data from the total amygdala and each of its subnuclei groups (CeM, centromedial group; BA, basal group; LA, lateral nucleus). (b). Classification accuracy above median of achieved by linear SVM classifiers trained separately either on the CeM BOLD response amplitude or the CeM BOLD response latency. *FWE $p<0.05 ; * * \mathrm{FWE} p<0.01$. 
Figure 8. Human intra-amygdala functional connectivity is represented by partial correlation coefficients (CeM, centromedial group; BA, basal group; LA, lateral nucleus). All correlation coefficients were statistically significant and different from each other. 


\begin{tabular}{|c|c|c|c|c|c|}
\hline Study & $\begin{array}{l}\text { Resolution } \\
\left(\mathrm{mm}^{3}\right)\end{array}$ & Stimuli & $\begin{array}{l}\text { Other } \\
\text { Methodological } \\
\text { Details }\end{array}$ & Summary of Results & $\begin{array}{c}\text { ROI vs. } \\
\text { Localization }\end{array}$ \\
\hline $\begin{array}{l}\text { Bach et al. } \\
\quad(2011)\end{array}$ & $1.5 \times 1.5 \times 1.5$ & electric shocks & N/A & $\begin{array}{l}\text { BLA group and centrocortical AG have } \\
\text { similar sensitivity profiles in fear conditioning }\end{array}$ & ROI \\
\hline $\begin{array}{l}\text { Boll et al. } \\
(2011)\end{array}$ & $1.5 \times 1.5 \times 1.5$ & $\begin{array}{l}\text { angry and fearful } \\
\text { faces }\end{array}$ & $\begin{array}{l}\text { Manipulated context } \\
\text { as self vs. other as } \\
\text { cause of facial } \\
\text { expression }\end{array}$ & $\begin{array}{l}\text { AB nucleus responded to facial expression in } \\
\text { general, regardless of the stimulus context; } \\
\text { context-dependent threat evaluation was } \\
\text { localized to the corticomedial AG }\end{array}$ & Cluster \\
\hline $\begin{array}{l}\text { Boll et al. } \\
(2013)\end{array}$ & $1.5 \times 1.5 \times 1.5$ & electric shocks & $\begin{array}{l}\text { Employed Pavlovian } \\
\text { conditioning } \\
\text { procedure with cue } \\
\text { reversal }\end{array}$ & $\begin{array}{l}\text { Corticomedial activity was positively } \\
\text { correlated with outcome uncertainty; BLA } \\
\text { activity was correlated with outcome certainty }\end{array}$ & Cluster \\
\hline $\begin{array}{l}\text { Gamer et al. } \\
\text { (2010) }\end{array}$ & $2.0 \times 2.0 \times 2.0$ & $\begin{array}{l}\text { happy, neutral, } \\
\text { and fearful faces }\end{array}$ & $\begin{array}{l}\text { Administering } \\
\text { oxytocin or placebo }\end{array}$ & $\begin{array}{l}\text { Oxytocin reduced sensitivity of dorsal and } \\
\text { lateral AG to fearful faces, while enhancing } \\
\text { sensitivity to happy faces }\end{array}$ & Cluster \\
\hline $\begin{array}{l}\text { Frühholz and } \\
\text { Grandjean } \\
\quad(2013)\end{array}$ & $1.5 \times 1.5 \times 2.4$ & $\begin{array}{l}\text { nonwords spoken } \\
\text { in angry or neutral } \\
\text { tone }\end{array}$ & $\mathrm{N} / \mathrm{A}$ & $\begin{array}{l}\text { Superficial complex and the LB complex } \\
\text { were sensitive to emotional tone; activity was } \\
\text { modulated by attentional focus and proximal } \\
\text { temporal context }\end{array}$ & Cluster \\
\hline $\begin{array}{l}\text { Prévost et al. } \\
\qquad(2011)\end{array}$ & $1.58 \times 1.63 \times 2.5$ & $\begin{array}{l}\text { monetary } \\
\text { outcomes }\end{array}$ & $\begin{array}{l}\text { Manipulated context } \\
\text { as reward, } \\
\text { punishment, or } \\
\text { neutral in a choice } \\
\text { task }\end{array}$ & $\begin{array}{l}\text { BLA showed greater activation for successful } \\
\text { attainment of reward; CeM showed greater } \\
\text { activation for successful avoidance of } \\
\text { punishments }\end{array}$ & $\begin{array}{l}\text { Cluster within } \\
\text { anatomically } \\
\text { defined ROIs }\end{array}$ \\
\hline $\begin{array}{l}\text { Prévost et al. } \\
\qquad(2012)\end{array}$ & $1.8 \times 1.8 \times 1.8$ & $\begin{array}{l}\text { food images and } \\
\text { rewards }\end{array}$ & $\begin{array}{l}\text { General vs. specific } \\
\text { Pavlovian-to- } \\
\text { instrumental transfer } \\
\text { (PIT) }\end{array}$ & $\begin{array}{l}\text { Activity in CeM correlated with general PIT } \\
\text { across participants; BLA correlated with } \\
\text { specific PIT }\end{array}$ & $\begin{array}{l}\text { Cluster within } \\
\text { anatomically } \\
\text { defined ROIs }\end{array}$ \\
\hline $\begin{array}{l}\text { Prévost et al. } \\
\qquad(2013)\end{array}$ & $1.58 \times 1.63 \times 2.5$ & $\begin{array}{l}\text { Pleasant, neutral, } \\
\text { and unpleasant } \\
\text { liquids }\end{array}$ & $\begin{array}{l}\text { Appetitive vs. } \\
\text { aversive Pavlovian } \\
\text { learning }\end{array}$ & $\begin{array}{l}\text { Investigated model-based learning in the } \\
\text { amygdala: expected value signals in BLA } \\
\text { correlated with in appetitive learning, while } \\
\text { expected value signals in CeM activity } \\
\text { correlated with aversive learning; precision } \\
\text { signals in CeM correlated with precision } \\
\text { signals in both types of learning }\end{array}$ & $\begin{array}{l}\text { Cluster within } \\
\text { anatomically } \\
\text { defined ROIs }\end{array}$ \\
\hline
\end{tabular}




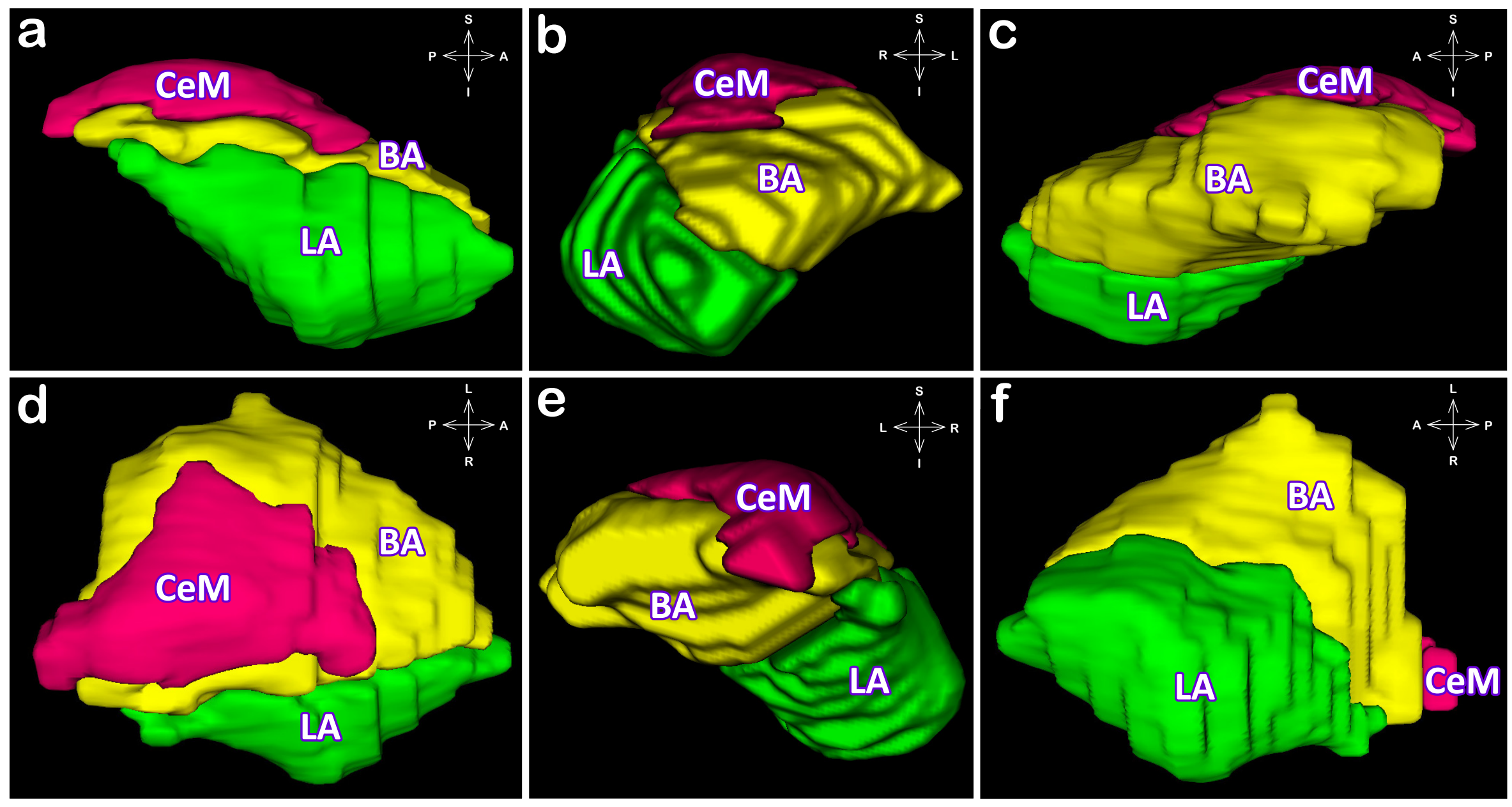



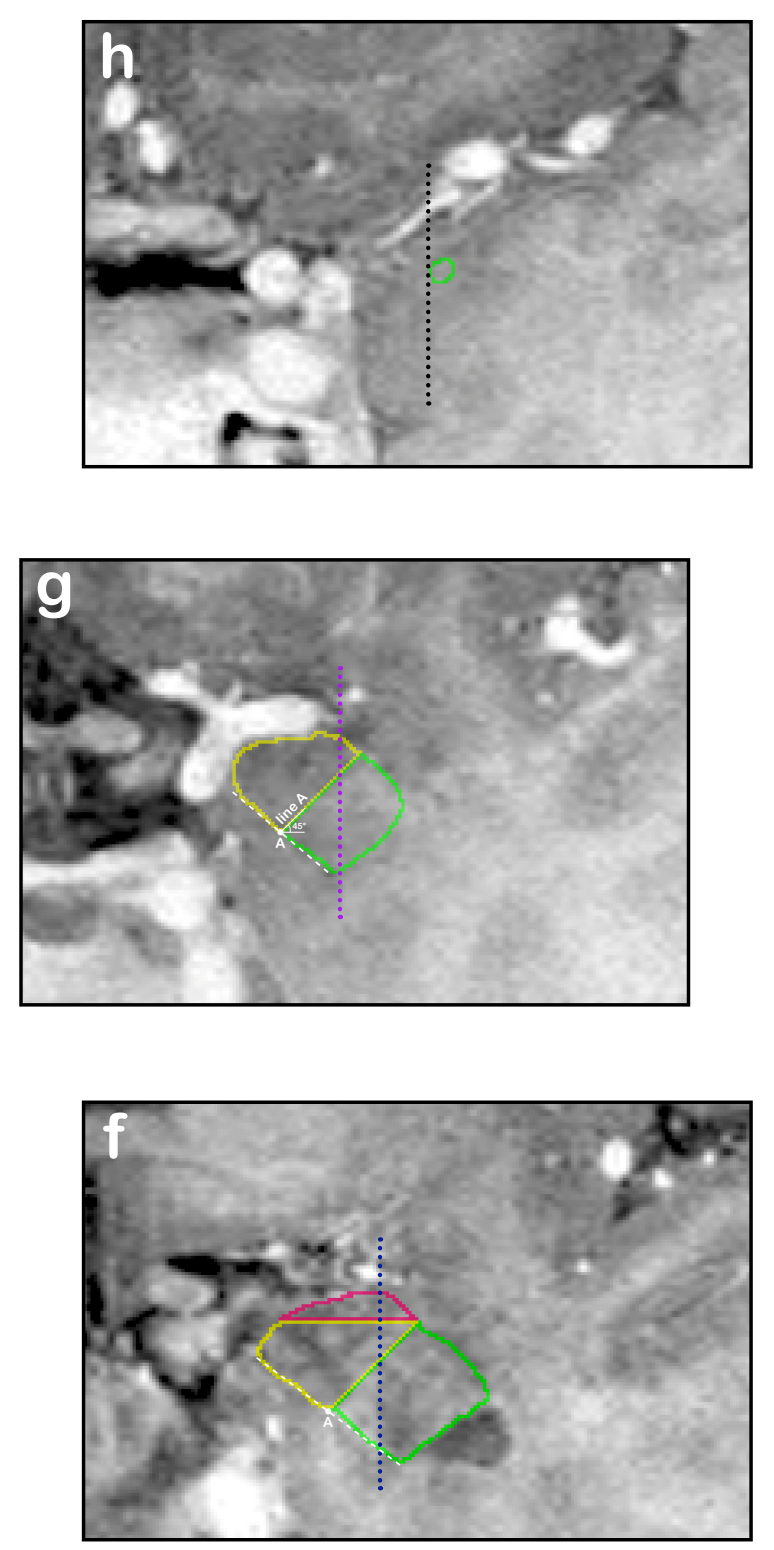
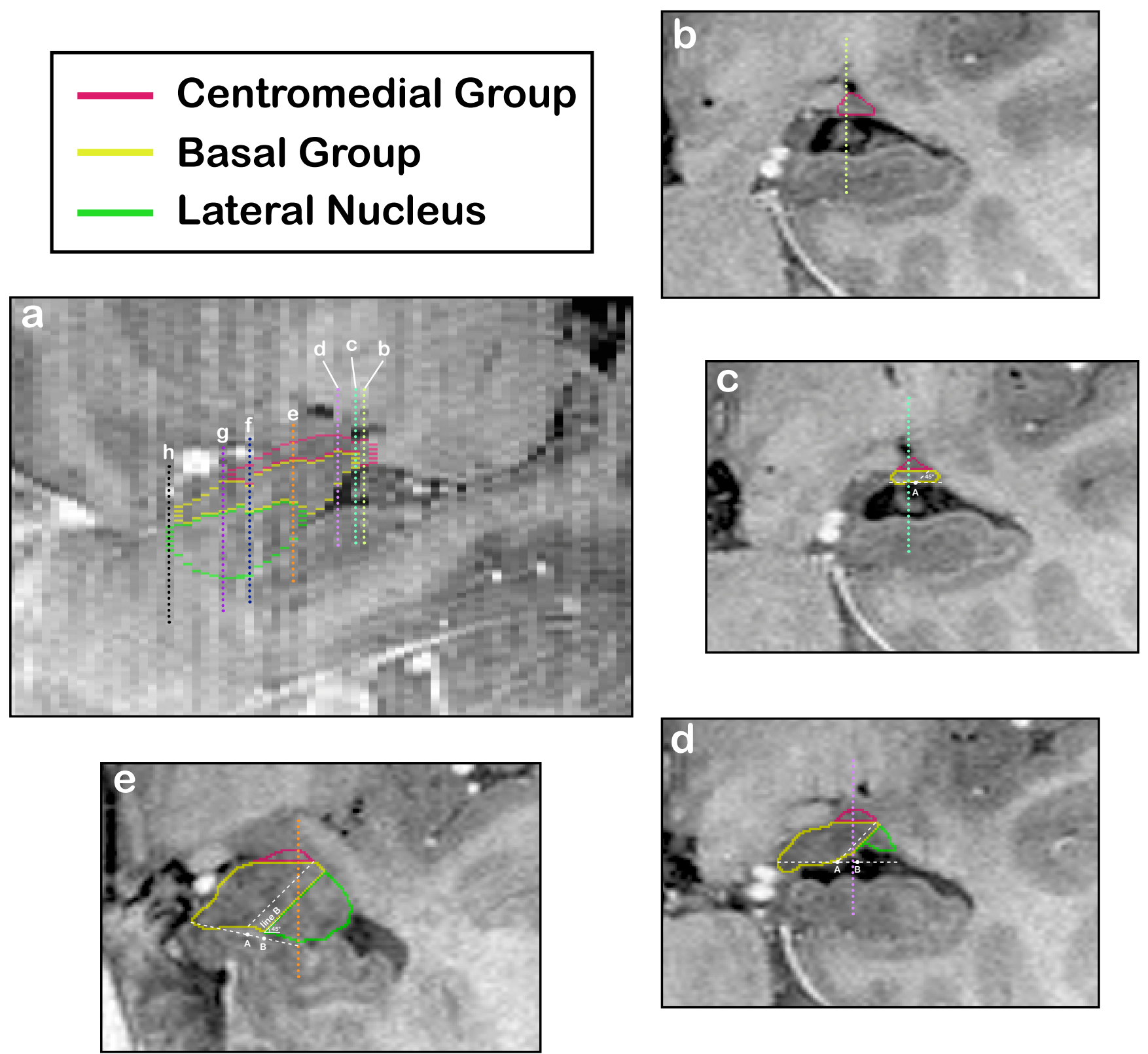


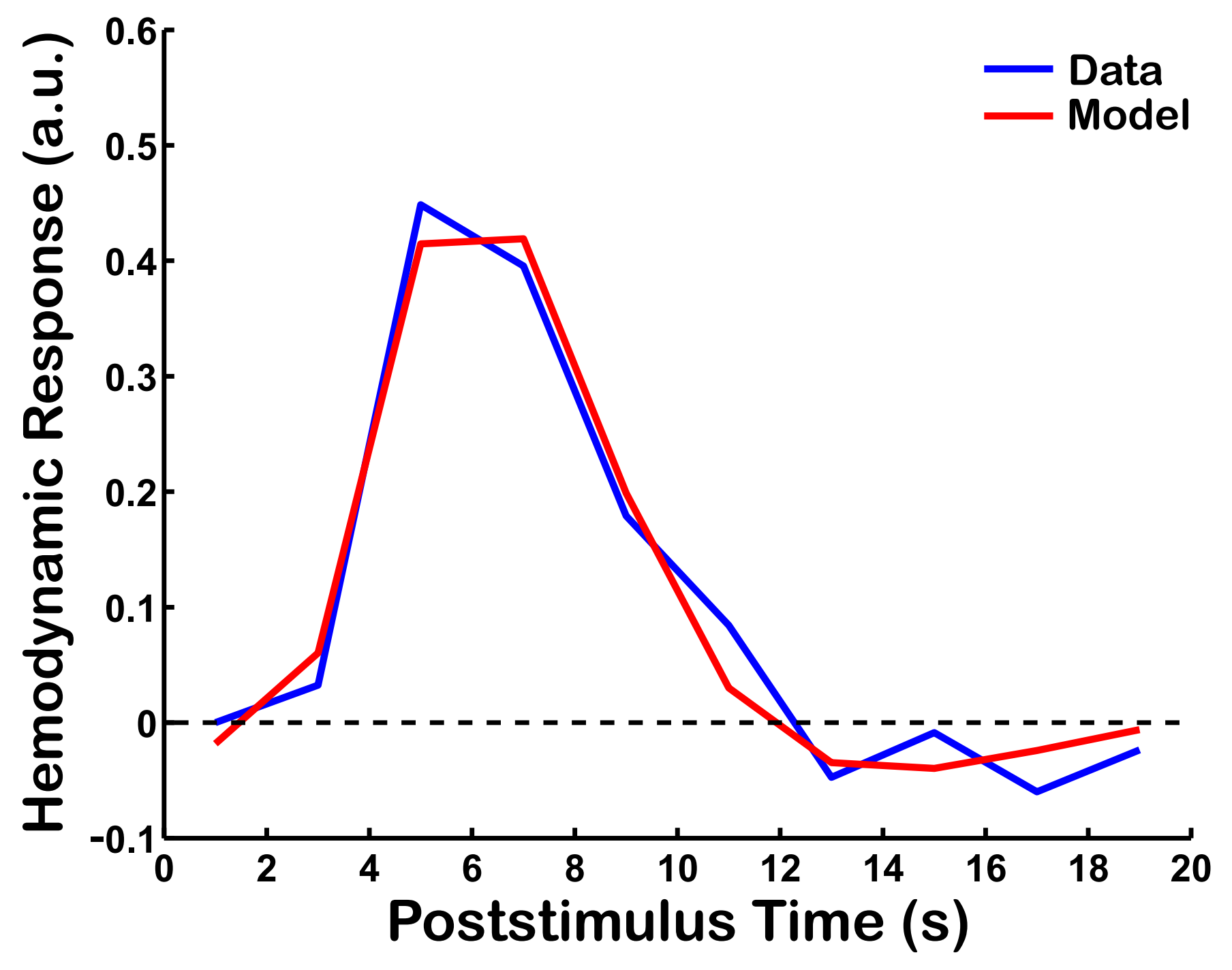



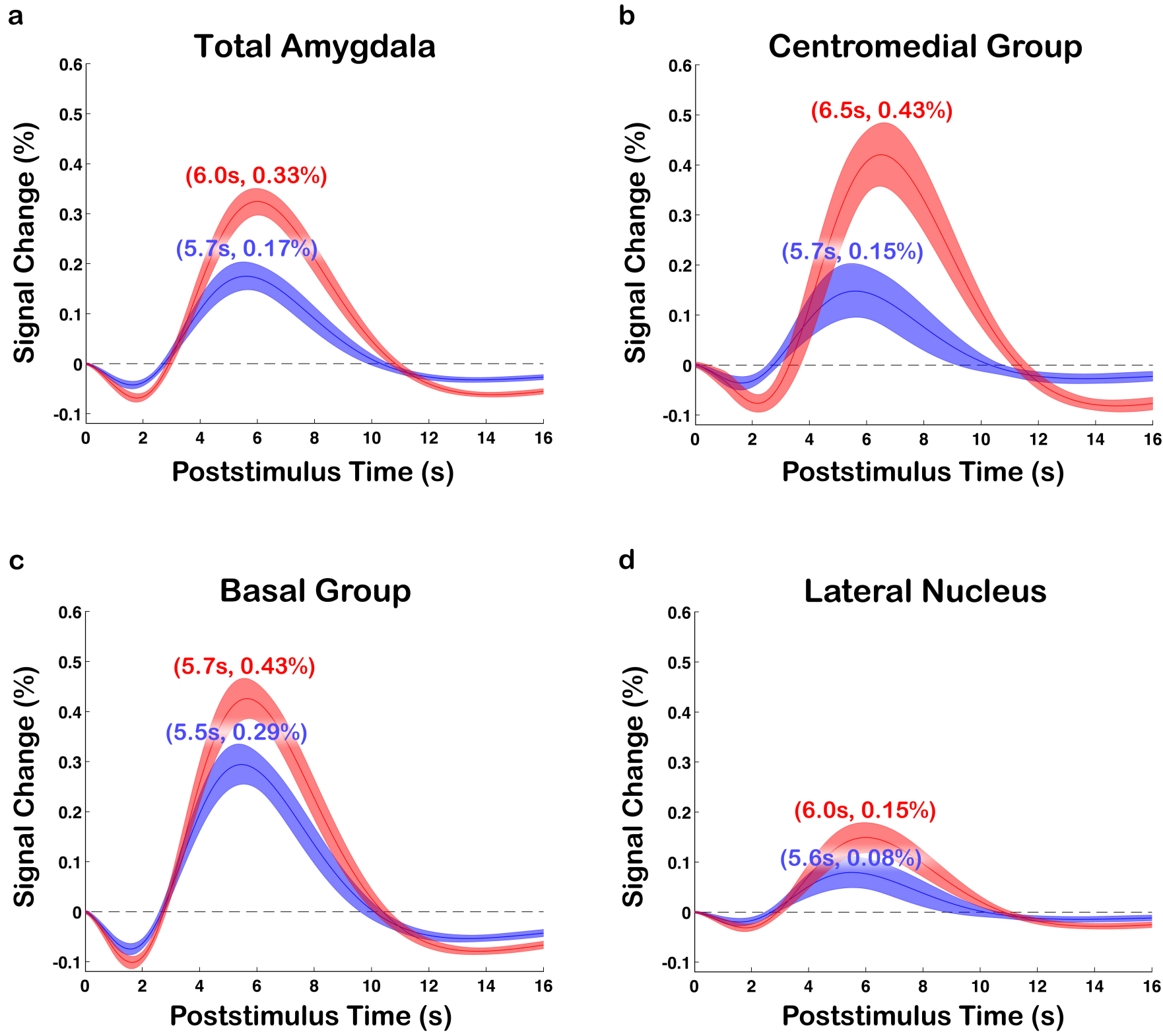
a

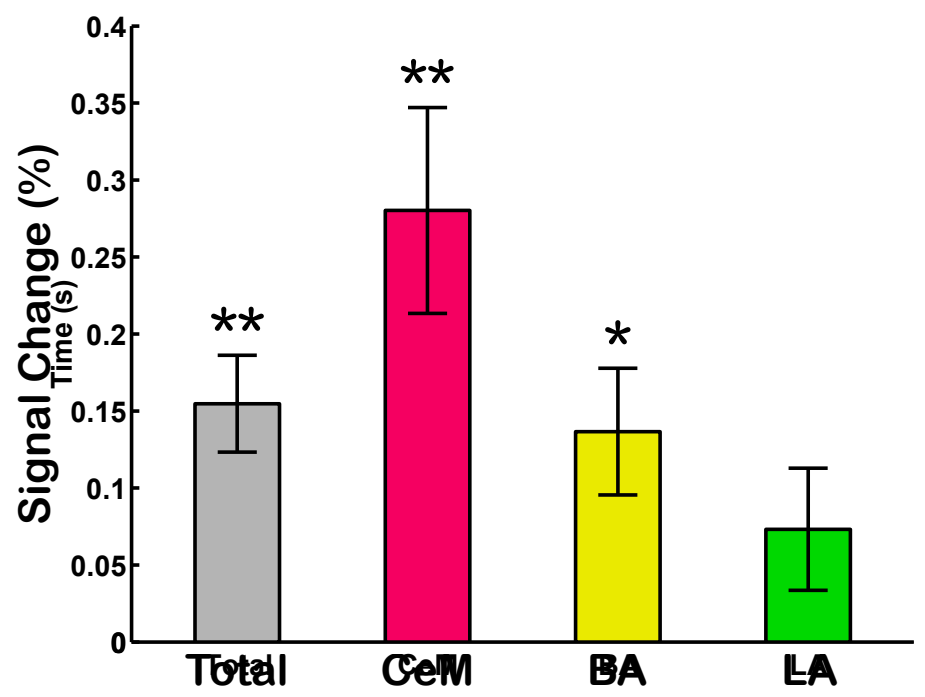

b

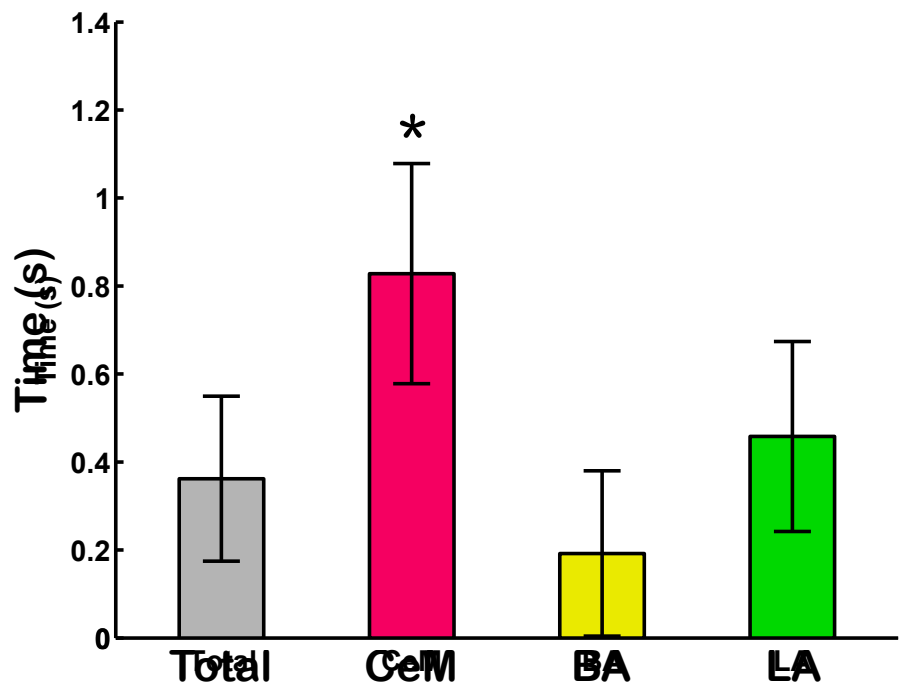


a

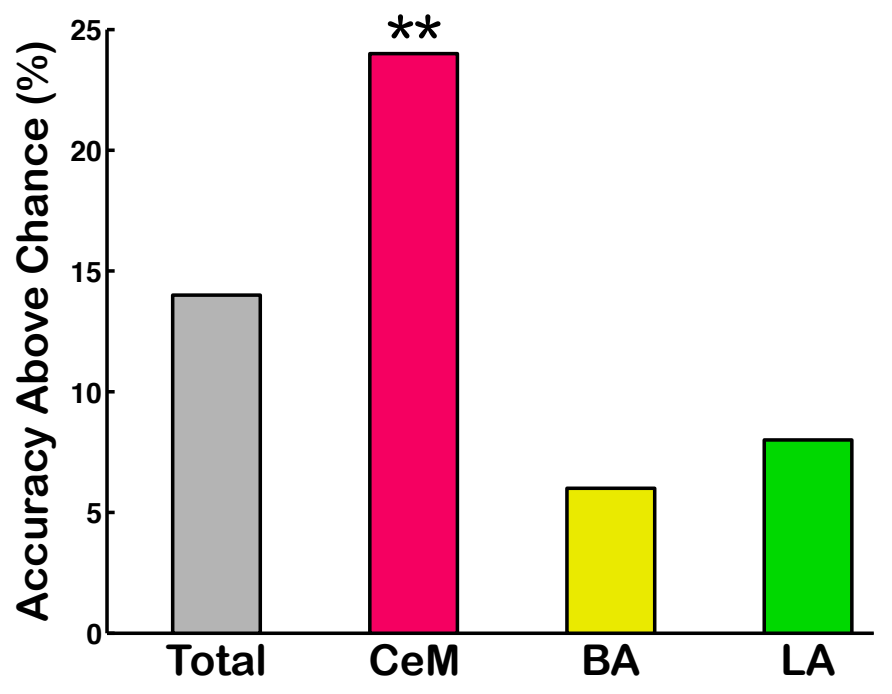

b

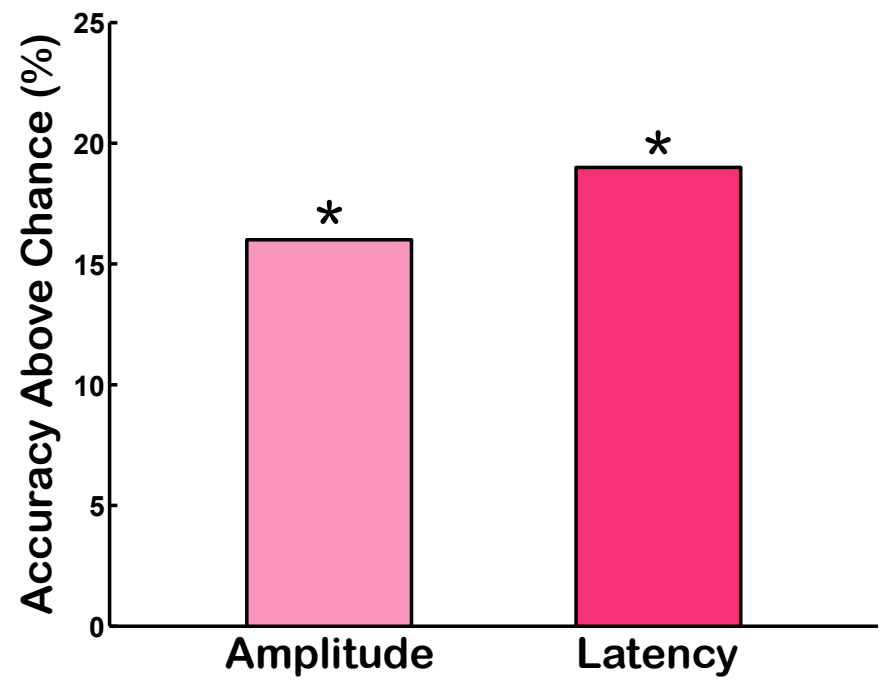




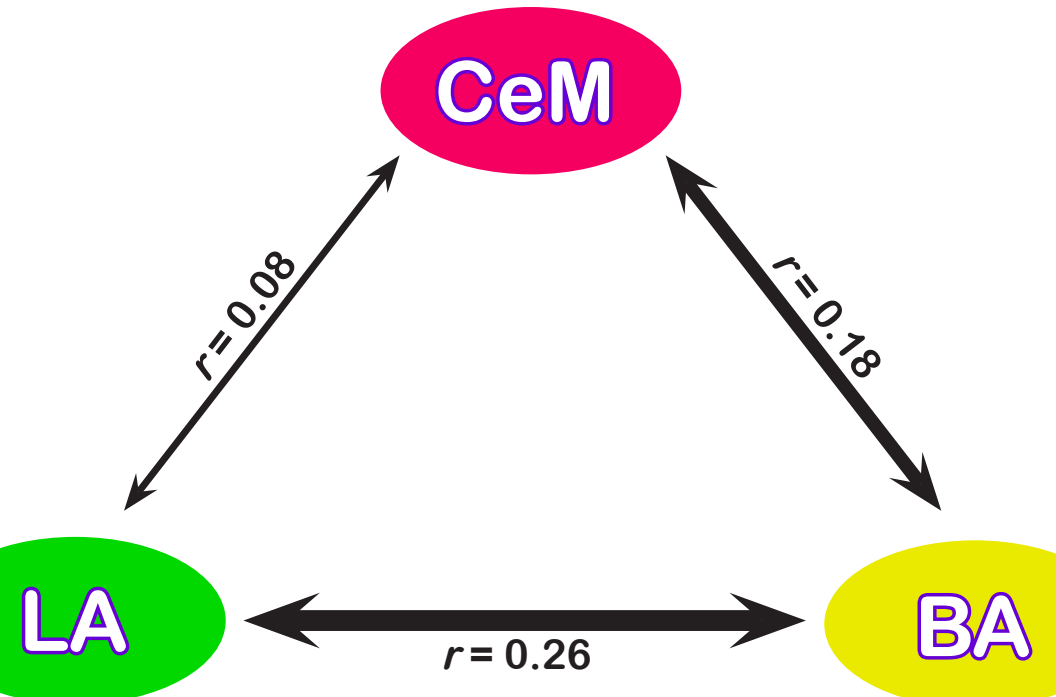

